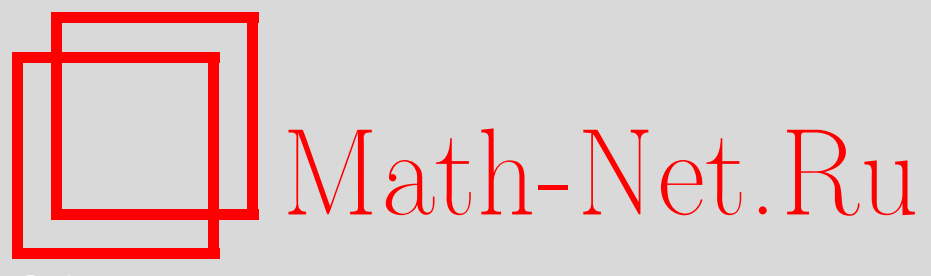

А. А. Доси, Некоммутативные голоморфные функции от элементов алгебры Ли и задача об абсолютном базисе, Изв. РАН. Сер. матем., 2009, том 73, выпуск 6, 77-100

DOI: https://doi.org/10.4213/im2661

Использование Общероссийского математического портала Math-Net.Ru подразумевает, что вы прочитали и согласны с пользовательским соглашением http://www.mathnet.ru/rus/agreement

Параметры загрузки:

IP : 44.207 .124 .84

26 апреля 2023 г., 16:17:53

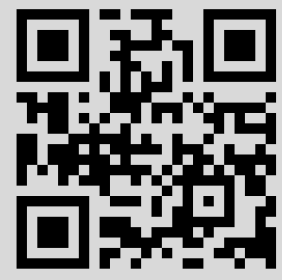




\title{
А. А. Доси \\ Некоммутативные голоморфные функции от элементов алгебры Ли и задача об абсолютном базисе
}

\begin{abstract}
Изучается задача об абсолютном базисе в алгебрах голоморфных функций от некоммутирующих переменных, порождающих конечномерную нильпотентную алгебру Ли g. Постановка этой задачи мотивирована программой Дж. Тэйлора о построении некоммутативного голоморфного функционального исчисления в контексте алгебр Ли.

Библиография: 17 наименований.
\end{abstract}

Ключевые слова: голоморфные функции от элементов алгебры Ли, оболочка Аренса-Майкла, локализация.

\section{$\S 1$. Введение}

Основными компонентами некоммутативного голоморфного функционального исчисления (см. [1]-[3]) являются:

1) основная алгебра "полиномов" $\mathcal{B}$;

2) банахов левый $\mathcal{B}$-модуль $X$;

3) семейство топологических алгебр $\mathcal{A}_{\kappa}$ "функций" с гомоморфизмами $\iota_{\kappa}$ : $\mathcal{B} \rightarrow \mathcal{A}_{\kappa}$.

Задача построения некоммутативного голоморфного функционального исчисления состоит в нахождении таких алгебр $\mathcal{A}_{\kappa}$, чтобы банахово пространство $X$ было левым $\mathcal{A}_{\kappa}$-модулем, для которого новая структура $\mathcal{B}$-модуля, полученная переносом с помощью гомоморфизма $\iota_{\kappa}$, сводилась бы к исходной. Задача о функциональном исчислении от элементов набора $T=\left(T_{1}, \ldots, T_{n}\right)$ из $n$ попарно коммутирующих операторов на банаховом пространстве $X$ (см. [4], [5]) переформулируется в этих терминах следующим образом: в качестве основной алгебры $\mathcal{B}=P_{n}$ берется алгебра всех полиномов от $n$ коммутирующих переменных $z=\left(z_{1}, \ldots, z_{n}\right)$, пространство $X$ снабжается естественной структурой $\mathcal{B}$-модуля по формуле $(p(z), x) \mapsto p(T) x$, а в качестве $\mathcal{A}_{\kappa}$ берутся алгебры $\mathcal{O}(D)$ голоморфных функций на областях $D \subseteq \mathbb{C}^{n}$. Если основная алгебра $\mathcal{B}$ отлична от $P_{n}$, то возникает вопрос: как определить класс алгебр $\mathcal{A}_{\kappa}$ “функций от некоммутирующих переменных", который играл бы для $\mathcal{B}$ ту же роль, что и алгебры $\mathcal{O}(D)$ для $P_{n}$ ? Чтобы описать эту роль алгебр $\mathcal{A}_{\kappa}$ по отношению к основной алгебре $\mathcal{B}$, в работе [1] был в гомологических терминах определен класс гомоморфизмов топологических алгебр $\iota: \mathcal{B} \rightarrow \mathcal{A}$, называемых локализациями (см. также [6], [7]). Грубо говоря, гомоморфизм $\iota: \mathcal{B} \rightarrow \mathcal{A}$ является локализацией, если функторы гомологий Хохшильда устойчивы при переходе от $\mathcal{B}$ к $\mathcal{A}$ посредством $\iota$. Основным примером локализации для алгебры полиномов $\mathcal{B}=P_{n}$ служит каноническое вложение $P_{n} \rightarrow \mathcal{O}(D)$, где $D-$ область голоморфности. 
Некоторые некоммутативные версии алгебр голоморфных функций на полидисках (т. е. претендентов на роль алгебр $\mathcal{A}_{\kappa}$ ) рассматривались в [8], [9]. Пусть $\mathfrak{g}$ - нильпотентная алгебра Ли, $\mathcal{U}(\mathfrak{g})$ - ее универсальная обертывающая алгебpa, а $D=D_{1} \times \cdots \times D_{n}-$ полидиск в $\mathbb{C}^{n}, n=\operatorname{dim}(\mathfrak{g})$. Алгебра $\mathcal{O}_{\mathfrak{g}}(D)$ всех голоморфных функиий (на $D$ ) от элементов алгебры $\mathfrak{g}$ определяется как пополнение алгебры $\mathcal{U}(\mathfrak{g})$ по некоторой системе полунорм. Если алгебра Ли $\mathfrak{g}$ коммутативна, то $\mathcal{O}_{\mathfrak{g}}(D)$ сводится к полупростой алгебре $\mathcal{O}(D)$ всех обычных голоморфных функций на $D$. В случае $D=\mathbb{C}^{n}$ алгебра $\mathcal{O}_{\mathfrak{g}}(D)$ обозначается через $\mathcal{O}_{\mathfrak{g}}$ и называется алгеброй всех целых функиий от элементов алгеб$p \mathfrak{g} \mathfrak{g}$. Если $\mathfrak{g}$ - некоммутативная нильпотентная алгебра Ли, то алгебра $\mathcal{O}_{\mathfrak{g}}(D)$ имеет нетривиальный радикал Джекобсона $\operatorname{Rad} \mathcal{O}_{\mathfrak{g}}(D)$ (это замкнутый идеал, порожденный идеалом Ли $[\mathfrak{g}, \mathfrak{g}])$ и факторалгебра $\mathcal{O}_{\mathfrak{g}}(D) / \operatorname{Rad} \mathcal{O}_{\mathfrak{g}}(D)$ сводится к алгебре $\mathcal{O}_{\mathfrak{g} /[\mathfrak{g}, \mathfrak{g}]}\left(D_{*}\right)\left(=\mathcal{O}\left(D_{*}\right)\right)$ обычных голоморфных функций на суженном полидиске $D_{*}=D_{1} \times \cdots \times D_{m}, m=\operatorname{dim}(\mathfrak{g} /[\mathfrak{g}, \mathfrak{g}])$ [9]. Возникает вопрос: будет ли вложение $\mathcal{U}(\mathfrak{g}) \rightarrow \mathcal{O}_{\mathfrak{g}}(D)$ локализацией? В случае положительного ответа мы получаем семейство некоммутативных алгебр $\mathcal{A}_{\kappa}$ для построения "нильпотентного функционального исчисления" в подходе Тэйлора. Как показано в [10], [11], эта задача о локализации имеет прямое отношение к свойству деления в $\mathcal{O}_{\mathfrak{g}}(D)$, которое, в свою очередь, связано с существованием абсолютного базиса в $\mathcal{O}_{\mathfrak{g}}(D)$. А именно, пусть $e=\left(e_{1}, \ldots, e_{n}\right)$ - базис нильпотентной алгебры Ли $\mathfrak{g}$, подчиненный нижнему центральному ряду этой алгебры. Будем называть е треугольным базисом. В работе [9] показано, что для каждого $f \in \mathcal{O}_{\mathfrak{g}}(D)$ существует единственный набор $a_{J}$ комплексных чисел такой, что $f$ можно записать в виде формального степенного ряда: $f=\sum_{J} a_{J} e^{J}$ (тейлоровское разложение), где $e^{J}=e_{1}^{j_{1}} \cdots e_{n}^{j_{n}}-$ упорядоченный моном по базису $e$ в $\mathcal{U}(\mathfrak{g})$. Сходимость этого ряда понимается в специальном смысле, называемом (слабой) однородной сходимостъю (см. п. 2.1), и в этом смысле можно сказать, что множество $\left\{e^{J}\right\}$ всех упорядоченных мономов является (слабым) однородным базисом в $\mathcal{O}_{\mathfrak{g}}(D)$. Таким образом, мы получаем некоммутативный аналог классического (тейлоровского) разложения обычных голоморфных функций нескольких комплексных переменных.

Основной задачей, изучаемой в настоящей работе, является задача об абсолютном базисе в $\mathcal{O}_{\mathfrak{g}}(D)$ : будет ли множество $\left\{e^{J}\right\}$ всех упорядоченных мономов в $\mathcal{U}(\mathfrak{g})$ абсолютным (или безусловным) базисом в $\mathcal{O}_{\mathfrak{g}}(D)$, т. е. верно ли, что разложение любого $f \in \mathcal{O}_{\mathfrak{g}}(D)$ в степенной ряд $\sum_{J} a_{J} e^{J}$ сходится абсолютно в $\mathcal{O}_{\mathfrak{g}}(D)$, как это было в коммутативном случае? Решение дается в терминах понятия роста (см. ниже определение 3.1 ) треугольного базиса $е$ для случая, когда $\mathcal{O}_{\mathfrak{g}}(D)=\mathcal{O}_{\mathfrak{g}}$ - алгебра всех целых функций от элементов алгебры $\mathfrak{g}$. А именно, если треугольный базис $e$ имеет нормальный рост, то $\left\{e^{J}\right\} \subseteq \mathcal{U}(\mathfrak{g})$ является абсолютным базисом в $\mathcal{O}_{\mathfrak{g}}($ см. $\S 4)$. Этот результат позволяет нам дать полное описание топологии $\mathcal{O}_{\mathfrak{g}}$ явно заданным семейством норм (см. п. 4.3). Наконец, мы покажем, что $\left\{e^{J}\right\}$ - абсолютный базис в $\mathcal{O}_{\mathfrak{g}}(D)$, но локально, для ограниченного полидиска $D$. Некоторые из результатов статьи были анонсированы в [10].

Наконец, я поблагодарю Ю. В. Туровского и А. Ю. Пирковского за ценные замечания и предложения. Автор также благодарен рецензенту за предложенные комментарии. 


\section{§ 2. Предварительные сведения}

Все рассматриваемые векторные пространства являются комплексными. Тождественный оператор на векторном пространстве $X$ обозначается через $1_{X}$. Если $X$ - нормированное пространство, то его норма обозначается через $\|\cdot\|_{X}$, а двойственное пространство - через $X^{*}$. Если $X$ - конечномерное векторное пространство с базисом $e=\left(e_{1}, \ldots, e_{n}\right)$, то через $l_{e}$ обозначается $\ell_{1}$-норма на $X$ относительно $e$, т. е. $l_{e}\left(\sum a_{i} e_{i}\right)=\sum\left|a_{i}\right|, a_{i} \in \mathbb{C}$. Пусть $\mathbb{R}_{+}^{n}-n$-я декартова степень множества $\mathbb{R}_{+}$всех положительных вещественных чисел, и пусть $\overline{\mathbb{R}}_{+}=\mathbb{R}_{+} \cup\{+\infty\}$. Для $s, t \in \mathbb{R}_{+}^{n}$ записываем $s \leqslant t$, если $s_{i} \leqslant t_{i}$ для всех $i$, $1 \leqslant i \leqslant n$, где $s_{i}=s(i), t_{i}=t(i)$. Ясно, что отношение $\leqslant$ превращает $\mathbb{R}_{+}^{n}$ в частично упорядоченное множество. Направленное подмножество $S \subseteq \mathbb{R}_{+}^{n}$ называется неограниченно возрастающим, если для любого числа $k \in \mathbb{N}$ найдется элемент $s^{(k)} \in S$ такой, что $\min \left(s^{(k)}\right) \geqslant k$. Будем для краткости заменять обозначения $\max \{a, b\}$ и $\min \{a, b\}$ на $a \vee b$ и $a \wedge b$ для $a, b \in \mathbb{R}$, если это не ведет к путанице. При $s \in \mathbb{R}_{+}^{n}$ обозначим $\ell_{1}$-норму на $X$ относительно базиса $s^{-1} e=\left(s_{1}^{-1} e_{1}, \ldots, s_{n}^{-1} e_{n}\right)$ через $l_{e, s}$. Векторное пространство всех непрерывных линейных операторов $X \rightarrow Y$ между локально выпуклыми пространствами $X$ и $Y$ обозначается через $\mathcal{L}(X, Y)$. При $X=Y$ мы записываем $\mathcal{L}(X)$ вместо $\mathcal{L}(X, X)$. Радикал Джекобсона ассоциативной алгебры $A$ обозначается через $\operatorname{Rad} A$. Алгебра всех $(n \times n)$-матриц над алгеброй $A$ обозначается через $\mathcal{M}_{n}(A)$. Операторы левого и правого умножения на $A$ обозначаются через $L_{a}$ и $R_{a}$, так что $L_{a}(x)=a x$ и $R_{a}(x)=x a$ для всех $a, x \in A$. Единица алгебры $A$ обозначается через $1_{A}$.

Пусть $F$ - конечномерная алгебра Ли. Ее нижним иентральным рядом называется последовательность идеалов $F^{(k)}, k \in \mathbb{N}$, где $F^{(1)}=F$ и $F^{(k)}=\left[F, F^{(k-1)}\right]$ при $k \geqslant 2$. Алгебра Ли $F$ называется нильпотентной, если $F^{(k)}=\{0\}$ для некоторого $k$. Если $F^{(k+1)}=\{0\}$, но $F^{(k)} \neq\{0\}$, то $k$ называется степенъю нильпотентности алгебры $F$. Нильпотентная алгебра Ли $F$ называется алгеброй Гейзенберга, если $\operatorname{dim}([F, F])=1$. В этом случае $F$ имеет степень нильпотентности 2. Простым примером алгебры Гейзенберга является алгебра Ли с базисом $e_{1}, e_{2}, e_{3}$ таким, что $\left[e_{1}, e_{2}\right]=e_{3}$ и $\left[e_{i}, e_{3}\right]=0$ для всех $i$.

Пространство всех характеров Ли алгебры Ли $F$ обозначается через $\Delta(F)$. Универсальная обертывающая алгебра алгебры Ли $F$ обозначается через $\mathcal{U}(F)$ и рассматривается как локально выпуклая алгебра с тончайшей локально выпуклой топологией. Пополнением обертывающей алгебры $\mathcal{U}(F)$ называется такая локально выпуклая алгебра $A$, которая содержит $\mathcal{U}(F)$ в качестве плотной подалгебры. Такое пополнение $A$ называется окаймленным, если канонический характер $\varepsilon_{F}: \mathcal{U}(F) \rightarrow \mathbb{C}, \varepsilon_{F}(F)=\{0\}$, непрерывен в топологии, индуцированной с $A$. Тем самым, $\varepsilon_{F}$ допускает продолжение до непрерывного характера $\varepsilon_{F}: A \rightarrow \mathbb{C}$. В этом случае удобно заменять обозначение $\varepsilon_{F}(f)$ на $f(0)$ для $f \in A$.

Лемма 2.1. Пусть $F-$ алгебра Ли, $u, v \in F u T_{v}=\operatorname{ad}(v) \in \mathcal{L}(\mathcal{U}(F))-$ оператор присоединенного представления. Тогда

$$
\left[u, v^{n}\right]=\sum_{k=1}^{n}(-1)^{k}\left(\left(\begin{array}{l}
n \\
k
\end{array}\right)-\left(\begin{array}{l}
n-4 \\
k-3
\end{array}\right)\right) v^{n-k} T_{v}^{k}(u) .
$$


ДокАЗАТЕЛЬСтво. Поскольку эта формула очевидно верна при $n=1$, можно воспользоваться индукцией по $n$. Из предположения индукции выводим, что

$$
\begin{aligned}
{\left[u, v^{n}\right]=} & -v^{n-1} T_{v}(u)+\left[u, v^{n-1}\right] v \\
= & -v^{n-1} T_{v}(u)+\sum_{k=1}^{n-1}(-1)^{k}\left(\left(\begin{array}{c}
n-1 \\
k
\end{array}\right)-\left(\begin{array}{c}
n-5 \\
k-3
\end{array}\right)\right) v^{n-k-1} T_{v}^{k}(u) v \\
= & \sum_{k=1}^{n-1}(-1)^{k}\left(\left(\begin{array}{c}
n-1 \\
k
\end{array}\right)-\left(\begin{array}{c}
n-5 \\
k-3
\end{array}\right)\right) v^{n-k} T_{v}^{k}(u) \\
& +\sum_{k=2}^{n}(-1)^{k}\left(\left(\begin{array}{c}
n-1 \\
k-1
\end{array}\right)-\left(\begin{array}{l}
n-5 \\
k-4
\end{array}\right)\right) v^{n-k} T_{v}^{k}(u)-v^{n-1} T_{v}(u) \\
= & -n v^{n-1} T_{v}(u)+\sum_{k=2}^{n}(-1)^{k}\left(\left(\begin{array}{l}
n \\
k
\end{array}\right)-\left(\left(\begin{array}{l}
n-5 \\
k-3
\end{array}\right)+\left(\begin{array}{l}
n-5 \\
k-4
\end{array}\right)\right)\right) v^{n-k} T_{v}^{k}(u) \\
= & \sum_{k=1}^{n}(-1)^{k}\left(\left(\begin{array}{l}
n \\
k
\end{array}\right)-\left(\begin{array}{l}
n-4 \\
k-3
\end{array}\right)\right) v^{n-k} T_{v}^{k}(u),
\end{aligned}
$$

откуда вытекает требуемая формула. Лемма доказана.

Пусть теперь $\mathfrak{g}$ - нильпотентная конечномерная алгебра Ли, и пусть $e=$ $\left(e_{1}, \ldots, e_{n}\right)$ - базис в $\mathfrak{g}$, подчиненный нижнему центральному ряду алгебры $\mathfrak{g}$. Это означает, что операторы присоединенного представления алгебры $\mathfrak{g}$ изображаются в базисе $е$ строго нижнетреугольными матрицами. В частности, для структурных констант $C_{i j}^{k}$ алгебры $\mathfrak{g}$ в базисе $e$ имеем $C_{i j}^{k}=0$ при $k \leqslant \max \{i, j\}$. Мы называем e треугольным базисом алгебры $\mathfrak{g}$. Если алгебра $\mathfrak{g}=\mathfrak{g}_{1} \oplus \cdots \oplus \mathfrak{g}_{c}$ градуирована натуральными числами $1, \ldots, c$, то любой базис, подчиненный этому разложению (и называемый градуированным базисом), треуголен. Ясно, что канонический базис алгебры Гейзенберга треуголен. Заметим, что если $e=\left(e_{1}, \ldots, e_{n}\right)$ - треугольный базис в $\mathfrak{g}$, то при некотором $m$ набор $e_{\mathrm{r}}=\left(e_{m+1}, \ldots, e_{n}\right)$ является базисом в $[\mathfrak{g}, \mathfrak{g}]$. Назовем набор $e_{\mathrm{r}}$ радикальной частью базиса $e$, а набор $e_{\mathrm{s}}=\left(e_{1}, \ldots, e_{m}\right)-$ полупростой частъю базиса $е$. Введем также обозначения $J_{\mathrm{s}}=\left(j_{1}, \ldots, j_{m}\right), J_{\mathrm{r}}=\left(j_{m+1}, \ldots, j_{n}\right)$ и $|J|=j_{1}+\cdots+j_{n}$ для любого набора $J=\left(j_{1}, \ldots, j_{n}\right) \in \mathbb{Z}_{+}^{n}$.

2.1. Однородный базис в пополнении $\mathcal{U}(\mathfrak{g})$. Пусть $\mathfrak{g}-$ конечномерная нильпотентная алгебра Ли, а $e=\left(e_{1}, \ldots, e_{n}\right)$ - ее треугольный базис. Введем понятие нильпотентной степени для (неупорядоченных) мономов $e_{i_{1}} \cdots e_{i_{s}}$ в $\mathcal{U}(\mathfrak{g})$ относительно базиса $e$, полагая $\operatorname{deg}\left(e_{i}\right)=\max \left\{k: e_{i} \in \mathfrak{g}^{(k)}\right\}$ для всех элементов $e_{i}$ базиса $e$ и $\operatorname{deg}(v)=\operatorname{deg}\left(e_{i_{1}}\right)+\cdots+\operatorname{deg}\left(e_{i_{s}}\right)$ для всех $v=e_{i_{1}} \cdots e_{i_{s}}$. Степень $\operatorname{deg}\left(e^{J}\right)$ упорядоченного монома $e^{J}$ будем также обозначать через $\langle J\rangle$. Обозначим через $\mathcal{U}_{k}(e)$ подпространство в $\mathcal{U}(\mathfrak{g})$, порожденное всеми упорядоченными мономами $e^{J}$ степени $k$, а через $\mathcal{U}^{k}(e)-$ подпространство, порожденное всеми мономами $e_{i_{1}} \cdots e_{i_{s}}$ степени не меньше $k$. Поскольку множество $\mathfrak{m}_{e}=\left\{e^{J}\right\}, e^{J}=e_{1}^{j_{1}} \cdots e_{n}^{j_{n}}, J \in \mathbb{Z}_{+}^{n}$, всех упорядоченных мономов есть алгебраический базис в $\mathcal{U}(\mathfrak{g})$, мы получаем, что $\mathcal{U}(\mathfrak{g})=\mathcal{U}_{0}(e) \oplus \cdots \oplus \mathcal{U}_{k-1}(e) \oplus \mathcal{U}^{k}(e)$ при каждом $k$. Заметим, что каждое подпространство $\mathcal{U}_{i}(e)$ конечномерно и имеет базис $\left\{e^{J}:\langle J\rangle=i\right\}$, тогда как $\mathcal{U}^{k}(e)=\bigoplus_{i \geqslant k} \mathcal{U}_{i}(e)$ (см. [9]). 
ОПРЕДЕЛЕНИЕ 2.1. Пусть $A$ - пополнение $\mathcal{U}(\mathfrak{g})$. Множество $\mathfrak{m}_{e}$ всех упорядоченных мономов от элементов треугольного базиса $е$ назовем слабо однороднъмм базисом в $A$, если при каждом $k$ имеем $A=\mathcal{U}_{0}(e) \oplus \cdots \oplus \mathcal{U}_{k-1}(e) \oplus \overline{\mathcal{U}^{k}(e)}$, где $\overline{\mathcal{U}^{k}(e)}$ - замыкание $\mathcal{U}^{k}(e)$ в $A$.

Таким образом, каждому $f \in A$ отвечает единственный набор комплексных чисел $\left\{a_{J}\right\}$ таких, что $f-\sum_{s=0}^{k-1} f_{s} \in \overline{\mathcal{U}^{k}(e)}$, где $f_{s}=\sum_{\langle J\rangle=s} a_{J} e^{J}$. Тем самым, имеется формальное разложение $f=\sum_{J} a_{J} e^{J}$. Если ряд $\sum_{k=0}^{\infty} f_{k}$ безусловно сходится к $f$ в $A$, то скажем, что ряд $\sum_{J} a_{J} e^{J}$ однородно сходится к $f$. Если это имеет место для всех $f \in A$, то подмножество $\mathfrak{m}_{e}$ называется однородным базисом в $A$. Аналогично, назовем $\mathfrak{m}_{e}$ абсолютным или безусловным базисом в $A$, если для каждого элемента $f \in A$ порожденный им ряд $\sum_{J} a_{J} e^{J}$ сходится к $f$ абсолютно или безусловно в $A$.

ЗАмЕчаниЕ 2.1. Пусть $A$ - пополнение универсальной обертывающей алгебры $\mathcal{U}(\mathfrak{g})$, снабженное слабо однородным базисом $\mathfrak{m}_{e}$. По определению 2.1 каждый элемент $f \in A$ имеет единственное формальное разложение: $f=$ $\sum_{J} a_{J} e^{J}$, так что определены координатные линейные функционалы $\alpha_{J}: A \rightarrow \mathbb{C}$, $\alpha_{J}(f)=a_{J}$. Пользуясь разложением из определения 2.1 , мы получаем, что все функционалы $\alpha_{J}$ непрерывны. Замкнутое подпространство $A_{0}=\bigcap_{J} \operatorname{ker}\left(\alpha_{J}\right)$ пространства $A$ состоит из всех элементов $f \in A$ с тривиальным формальным разложением. Ясно, что $A_{0}=\{0\}$ в том случае, когда $\left\{e^{J}\right\}$ является однородным базисом в $A$.

Некоторые пополнения $\mathcal{U}(\mathfrak{g})$ с однородными базисами рассматривались в [9]. Простым примером пополнения $\mathcal{U}(\mathfrak{g})$ с абсолютным базисом служит пространство $A=\mathcal{O}(D)$ голоморфных функций $n$ комплексных переменных. Для него $\mathfrak{g}=\mathbb{C}^{n}$ есть коммутативная алгебра Ли и каждый элемент $f \in \mathcal{O}(D)$ имеет классическое тейлоровское разложение: $f=\sum_{J} a_{J} z^{J}$, где $a_{J}=(J !)^{-1}\left(\partial^{|J|} f\right)(0)$. Здесь $\partial^{|J|}=\partial_{z_{1}}^{j_{1}} \cdots \partial_{z_{n}}^{j_{n}}, \partial_{z_{k}}-$ обычный оператор дифференцирования по комплексной переменной $z_{k}$, а $J=\left(j_{1}, \ldots, j_{n}\right) \in \mathbb{Z}_{+}^{n}$.

2.2. Оболочки Аренса-Майкла. Пусть $A$ - полная локально выпуклая алгебра. Если топология в $A$ задается семейством мультипликативных полунорм, то $A$ называется алгеброй Аренса-Майкла [12, п. 1.2.4]. Пусть теперь $A$ - локально выпуклая алгебра, умножение в которой раздельно непрерывно. Оболочкой Аренса-Майкла [12, п. 5.2.21] алгебры $A$ называется пара $(\tilde{A}, \omega)$, где $\tilde{A}$ - алгебра Аренса-Майкла, а $\omega: A \rightarrow \tilde{A}$ - непрерывный гомоморфизм алгебр, обладающий свойством "проективной универсальности": для любой алгебры Аренса-Майкла $B$ и любого непрерывного гомоморфизма алгебр $\pi: A \rightarrow B$ существует единственный непрерывный гомоморфизм алгебр $\tilde{\pi}: \tilde{A} \rightarrow B$ такой, что $\tilde{\pi} \cdot \omega=\pi$. Поскольку всякая алгебра Аренса-Майкла является обратным пределом банаховых алгебр [12, п. 5.2.10], достаточно проверять свойство проективной универсальности только для банаховых алгебр $B$.

Пусть $F$ - конечномерная алгебра Ли. Тогда оболочка Аренса-Майкла алгебры $\mathcal{U}(F)$ (снабженная тончайшей локально выпуклой топологией) обозначается через $\mathcal{O}_{F}$, а входящий в ее определение гомоморфизм - через $\omega_{F}$ : $\mathcal{U}(F) \rightarrow \mathcal{O}_{F}$. 
Лемма 2.2. Пусть $F$ - конечномерная алгебра Ли. Тогда $\operatorname{ker}\left(\omega_{F}\right)=\{0\}$ $u \mathcal{O}_{F}$ является окаймленным пополнением алгебры $\mathcal{U}(F)$. Кроме того, пространство всех непрерывных характеров алгебры $\mathcal{O}_{F}$ отождествляется с $\Delta(F)$.

ДоказАтельство. Пусть $p \in \mathcal{U}(F)$ - ненулевой элемент. Тогда существует представление $\pi: \mathcal{U}(F) \rightarrow A, \pi(p) \neq 0$, где $A$ - конечномерная банахова алгебpa $\left[13\right.$, п. 2.5.7]. Отсюда вытекает, что $\pi=\tilde{\pi} \cdot \omega_{F}$ для некоторого непрерывного гомоморфизма алгебр $\tilde{\pi}: \mathcal{O}_{F} \rightarrow A$, следовательно, $\widetilde{\pi}\left(\omega_{F}(p)\right)=\pi(p) \neq 0$. Тем самым, $\omega_{F}(p) \neq 0$. Поскольку в качестве $p$ можно было выбрать произвольный элемент $\mathcal{U}(F)$, мы заключаем, что $\operatorname{ker}\left(\omega_{F}\right)=\{0\}$. Таким образом, $\mathcal{U}(F)$ отождествляется с некоторой подалгеброй в $\mathcal{O}_{F}$. Пусть $\mathcal{B}$ - замыкание $\mathcal{U}(F)$ в $\mathcal{O}_{F}$. Тогда $\mathcal{B}$ является алгеброй Аренса-Майкла. Из единственности оболочки Аренса-Майкла получаем, что $\mathcal{B}=\mathcal{O}_{F}$. Остальные утверждения очевидны. Лемма доказана.

ЗАмечАниЕ 2.2. Пусть $\lambda \in \Delta(F)$, и пусть $F-\lambda=\{u-\lambda(u): u \in F\}$ - подалгебра Ли в $\mathcal{U}(F)$. Зададим линейное отображение $\psi: \mathcal{U}(F) \rightarrow \mathcal{O}_{F-\lambda}$ формулой $\psi(u)=(u-\lambda(u))+\lambda(u) 1_{\mathcal{O}_{F-\lambda}}, u \in F$. Тогда $\psi$ является гомоморфизмом алгебр и продолжается до изоморфизма топологических алгебр $\widehat{\psi}: \mathcal{O}_{F} \rightarrow \mathcal{O}_{F-\lambda}$, обратным к которому является продолжение гомоморфизма $\psi^{\prime}: \mathcal{U}(F-\lambda) \rightarrow \mathcal{O}_{F}$, $\psi^{\prime}(u-\lambda(u))=u-\lambda(u) 1_{\mathcal{O}_{F}}, u \in F$. По лемме 2.2 алгебра $\mathcal{O}_{F-\lambda}$ есть функциональное пополнение $\mathcal{U}(F-\lambda)$. Далее, если $\varepsilon_{F-\lambda}: \mathcal{O}_{F-\lambda} \rightarrow \mathbb{C}$ - тривиальный характер, то $\varepsilon_{F-\lambda} \cdot \widehat{\psi}=\lambda$. Действительно,

$$
\varepsilon_{F-\lambda}(\widehat{\psi}(u))=\varepsilon_{F-\lambda}\left((u-\lambda(u))+\lambda(u) 1_{\mathcal{O}_{F-\lambda}}\right)=\lambda(u)
$$

для всех $u \in F$. Таким образом, $\mathcal{O}_{F}$ есть окаймленное пополнение $\mathcal{U}(F-\lambda)$ с точностью до топологического изоморфизма.

Если $F$ - коммутативная алгебра Ли, то $\mathcal{O}_{F}$ сводится к алгебре всех целых функций от $n$ комплексных переменных, где $n=\operatorname{dim}(F)$ (см. $[12,5.2 .25]$ ), а $\varepsilon_{F}(f)$ есть не что иное, как значение функции $f$ в начале координат: $\varepsilon_{F}(f)=f(0)$.

\section{§ 3. Банахова обертывающая алгебра $\mathcal{A}(p)$}

Пусть $F$ - конечномерная алгебра Ли, а $p$ - норма на $F$. Следующее свойство проективной универсальности [8], [9] однозначно определяет банахову алгебру $\mathcal{A}(p)$ и гомоморфизм алгебр $\varphi_{F}: \mathcal{U}(F) \rightarrow \mathcal{A}(p)$ с плотным образом, причем $\left\|\varphi_{F} \mid F\right\| \leqslant 1$.

ЛЕмма 3.1. Для любой алгебры Ли $\mathcal{B}$ и гомоморфизма алгебр Ли $\alpha: F \rightarrow \mathcal{B}$, $\|\alpha\| \leqslant 1$, существует единственный гомоморфизм алгебр $\widetilde{\alpha}: \mathcal{A}(p) \rightarrow \mathcal{B},\|\widetilde{\alpha}\| \leqslant 1$, такой, что $\widetilde{\alpha} \cdot \varphi_{F}=\alpha$.

Алгебра $\mathcal{A}(p)$ называется (см. [9]) банаховой обертывающей алгеброй банаховой алгебры Ли $F$, снабженной нормой $p$.

Пусть теперь $\mathfrak{g}$ - нильпотентная алгебра Ли с нормой $p$. Тогда банахова обертывающая алгебра $\mathcal{A}(p)$ есть пополнение $\mathcal{U}(\mathfrak{g})$ по норме (см. [8], [9]). Следовательно, $\mathcal{A}(p)$ является окаймленным пополнением $\mathcal{U}(\mathfrak{g})$ согласно лемме 3.1 . 
Пусть $p=l_{e}$ есть $\ell_{1}$-норма (см. $\left.\S 2\right)$ на нильпотентной алгебре Ли $\mathfrak{g}$ относительно треугольного базиса $e=\left(e_{1}, \ldots, e_{n}\right)$ алгебры g. Семейство всех (неупорядоченных) мономов $v=e_{i_{1}} \cdots e_{i_{s}} \in \mathcal{U}(\mathfrak{g})$ от элементов базиса $e$ порождает $\mathcal{U}(\mathfrak{g})$ как векторное пространство, т. е. каждый элемент $u \in \mathcal{U}(\mathfrak{g})$ представляется линейной комбинацией этих мономов: $u=a_{1} v_{1}+\cdots+a_{k} v_{k}$. Для $u \in \mathcal{U}(\mathfrak{g})$ положим (см. [9])

$$
\|u\|=\inf \left\{\left|a_{1}\right|+\cdots+\left|a_{k}\right|: u=a_{1} v_{1}+\cdots+a_{k} v_{k}\right\},
$$

где точная нижняя грань берется по всем указанным представлениям элемента $u$. Поскольку $\mathfrak{g}$ - нильпотентная алгебра Ли, в (3.1) достаточно рассматривать только представления $u$ в виде линейной комбинации мономов, нильпотентные степени которых (см. п. 2.1) ограничены сверху некоторой константой, зависящей от $u$ и $n$. Поэтому равенство $\|u\|=0$ имеет место тогда и только тогда, когда $u=0$. Далее, $\mathcal{U}(\mathfrak{g})$ является нормированной алгеброй относительно нормы $\|\cdot\|$. Если $\mathfrak{A}-$ ее пополнение по норме и $\varphi: \mathcal{U}(\mathfrak{g}) \rightarrow \mathfrak{A}-$ каноническое вложение, то из (3.1) вытекает, что пара $(\mathfrak{A}, \varphi)$ обладает свойством проективной универсальности из леммы 3.1. Поэтому $\mathfrak{A}=\mathcal{A}\left(l_{e}\right)$ с точностью до изометрического изоморфизма.

Следующий результат был доказан в [9].

ПРЕДЛОЖЕНИЕ 3.1. Пусть $\mathfrak{g}$ - нилъпотентная алгебра Ли, a $l_{e}-\ell_{1}$-норма на $\mathfrak{g}$ относительно треугольного базиса е алгебры $\mathfrak{g}$. Тогда множество $\mathfrak{m}_{e} \subseteq \mathcal{U}(\mathfrak{g})$ всех упорядоченных мономов является слабо однородным базисом в $\mathcal{A}\left(l_{e}\right)$. Если алгебра $\mathfrak{g}$ градуирована, а е-градуированный базис в $\mathfrak{g}$, то $\mathfrak{m}_{e}$ является однородным базисом в $\mathcal{A}\left(l_{e}\right)$.

3.1. Норма координатного линейного функционала на $\mathcal{A}\left(l_{e}\right)$. Пусть $e=\left(e_{1}, \ldots, e_{n}\right)$ - треугольный базис нильпотентной алгебры Ли $\mathfrak{g}$, и пусть $\mathfrak{g}^{(c+1)}=\{0\}$. Тогда при некотором $m$ набор $e_{m+1}, \ldots, e_{n}$ является базисом в $[\mathfrak{g}, \mathfrak{g}]$. Кроме того, найдутся числа $d_{1}, \ldots, d_{c}$ такие, что наборы $\mathfrak{e}_{s}=$ $\left(e_{d_{s-1}+1}, \ldots, e_{d_{s}}\right)$ имеют одинаковую нильпотентную степень $s$, причем $d_{0}=0$, $d_{1}=m, d_{c}=n$. Фактически $\mathfrak{e}_{s}$ есть базис некоторого подпространства, дополняющего $\mathfrak{g}^{(s+1)}$ до $\mathfrak{g}^{(s)}$. Положим также $c^{*}=\max \left\{s: \operatorname{ad}^{s}\left(e_{j}\right)\left(e_{i}\right) \neq 0, i<j\right\}$. Ясно, что $c^{*} \leqslant c$. Пусть $e_{1}, \ldots, e_{m}$ - образующие Ли в $\mathfrak{g}$ нильпотентной степени 1 (тем самым, все $e_{i}$ не принадлежат $[\mathfrak{g}, \mathfrak{g}]$ ). Для каждого $s$ выберем максимальное линейно независимое подмножество всех лиевых слов $\left[e_{i_{1}},\left[e_{i_{2}}, \ldots\left[e_{i_{s-1}}, e_{i_{s}}\right] \ldots\right]\right]$ длины $s$ и нильпотентной степени $s$ и обозначим его через $\mathfrak{e}_{s}=\left(e_{d_{s-1}+1}, \ldots, e_{d_{s}}\right)$. Тогда $e=\mathfrak{e}_{1} \cup \cdots \cup \mathfrak{e}_{c}$ есть треугольный базис в $\mathfrak{g}$, называемый базисом, ассочиированным с образующими Ли $e_{1}, \ldots, e_{m}$. Для каждого набора $I=\left(i_{1}, \ldots, i_{n}\right) \in \mathbb{Z}_{+}^{n}$ и числа $k, 1 \leqslant k \leqslant c$, положим $I_{k}=\left(i_{d_{k-1}+1}, \ldots, i_{d_{k}}\right)$.

Лемма 3.2. Пусть $\mathfrak{g}$ - нильпотентная алгебра Ли с треуголъным базисом е, а $\alpha_{I} \in \mathcal{A}\left(l_{e}\right)^{*}$ - координатные линейные функциональ относительно слабо однородного базиса $\left\{e^{J}\right\}$ в $\mathcal{A}\left(l_{e}\right)$. Тогда

$$
\left\|\alpha_{I}\right\|_{\mathcal{A}\left(l_{e}\right)^{*}} \leqslant \varkappa^{c^{2}|I|} \prod_{\tau=1}^{c-1} \frac{\left(\left|I_{\tau}\right|+\sum_{\kappa=\tau+1}^{c} c^{*} \wedge(\kappa-\tau)\left|I_{\kappa}\right|\right) !}{\left|I_{\tau}\right| !},
$$


где $I=\left(i_{1}, \ldots, i_{n}\right) \in \mathbb{Z}_{+}^{n}, \varkappa=1 \vee\left(c\left(n-d_{1}\right) C\right), C=\sup \left\{\left|C_{i j}^{k}\right|: i, j, k\right\}, a\left\{C_{i j}^{k}\right\}-$ структурные константы алгебры $\mathfrak{g}$ относительно базиса е.

ДокАзАТЕЛЬСтво. Пусть $\theta_{I}-$ величина, стоящая в правой части неравенства, $e^{I}-$ базисный моном и $l=\operatorname{deg}\left(e^{I}\right)$. С учетом (3.1) достаточно доказать, что $\left|\alpha_{I}(v)\right| \leqslant \theta_{I}$ для всех (неупорядоченных) мономов $v$ от элементов базиса $e$. Фиксируем $1 \leqslant i, j \leqslant d_{c-1}$ и положим $T_{i}=\operatorname{ad}\left(e_{i}\right), T_{j}=\operatorname{ad}\left(e_{j}\right) \in \mathcal{L}(\mathcal{U}(\mathfrak{g}))$. Пусть $\mathcal{S}_{i j}$ - множество всех (неупорядоченных) мономов в $\mathcal{U}(\mathfrak{g})$ от элементов подмножества $\left\{e_{i}, e_{j}, T_{i}^{\varepsilon} T_{j}^{a}\left(e_{i}\right): a \geqslant 1, \varepsilon=0,1\right\}$ подалгебры Ли в $\mathfrak{g}$, порожденной элементами $e_{i}, e_{j}$. Для $v, w \in \mathcal{S}_{i j}$ запишем $v>w$, если моном $v$ имеет множитель $T_{j}^{a_{1}}\left(e_{i}\right) \cdots T_{j}^{a_{k}}\left(e_{i}\right)$ такой, что $k>s$ для каждого множителя $T_{j}^{b_{1}}\left(e_{i}\right) \cdots T_{j}^{b_{s}}\left(e_{i}\right)$ монома $w$. Ясно, что отношение $>$ можно продолжить до структуры линейного упорядочения на множестве $\mathcal{S}_{i j}$. Пользуясь леммой 2.1 и тем, что $\mathfrak{g}^{(c+1)}=\{0\}$, получаем равенство

$$
T_{i}\left(e_{j}^{p}\right)=\sum_{s=1}^{p}(-1)^{s}\left(\left(\begin{array}{l}
p \\
s
\end{array}\right)-\left(\begin{array}{c}
p-4 \\
s-3
\end{array}\right)\right) e_{j}^{p-s} T_{j}^{s}\left(e_{i}\right)=\sum_{s=1}^{c}(-1)^{s} \pi_{s}^{(p)} e_{j}^{p-s} T_{j}^{s}\left(e_{i}\right),
$$

где $\pi_{s}^{(p)}=\left(\begin{array}{l}p \\ s\end{array}\right)-\left(\begin{array}{l}p-4 \\ s-3\end{array}\right)$ при $s \leqslant p \leqslant c$ и $\pi_{s}^{(p)}=0$ при $c<s$. Фиксируем моном $e_{j}^{p} e_{i}^{q}$ и натуральное число $k, k \leqslant p \wedge q$. Покажем, что $e_{j}^{p} e_{i}^{q}$ можно записать в виде конечной линейной комбинации мономов из $\mathcal{S}_{i j}$ с выбранными "верхними" мономами, а именно

$$
e_{j}^{p} e_{i}^{q}=\sum_{s_{1}, \ldots, s_{k}=1}^{c} \lambda_{s_{1} \cdots s_{k}} v_{s_{1} \cdots s_{k}}+\sum_{v_{t}^{(k)} \in \mathcal{S}_{i j}} \lambda_{t}^{(k)} v_{t}^{(k)}
$$

причем $v_{s_{1} \cdots s_{k}}>\sup \left\{v_{t}^{(k)}: \lambda_{t}^{(k)} \neq 0\right\}$ и $\left|\lambda_{s_{1} \cdots s_{k}}\right| \geqslant \sup \left\{\left|\lambda_{t}^{(k)}\right|\right\}$ для всех $s_{1}, \ldots, s_{k}$, где

$$
\begin{aligned}
& v_{s_{1} \cdots s_{k}}=e_{j}^{p-\sum_{m=1}^{k} s_{m}} T_{j}^{s_{k}}\left(e_{i}\right) \cdots T_{j}^{s_{1}}\left(e_{i}\right) e_{i}^{q-k}, \\
& \lambda_{s_{1} \cdots s_{k}}=(-1)^{k+\sum_{m=1}^{k} s_{m}} \prod_{b=1}^{k} \pi_{s_{b}}^{\left(p-\sum_{m=1}^{b-1} s_{m}\right)} .
\end{aligned}
$$

Воспользуемся индукцией по $k$. При $k=1$ требуемое равенство вытекает из соотношения (3.2). Действительно,

$$
e_{j}^{p} e_{i}^{q}=-T_{i}\left(e_{j}^{p}\right) e_{i}^{q-1}+e_{i} e_{j}^{p} e_{i}^{q-1}=\sum_{s=1}^{c}(-1)^{s+1} \pi_{s}^{(p)} e_{j}^{p-s} T_{j}^{s}\left(e_{i}\right) e_{i}^{q-1}+e_{i} e_{j}^{p} e_{i}^{q-1}
$$

и $e_{j}^{p-s} T_{j}^{s}\left(e_{i}\right) e_{i}^{q-1}>e_{i} e_{j}^{p} e_{i}^{q-1}$ для всех $s$ по предположению. Заметим, что $\lambda_{s_{1}}=$ $(-1)^{s_{1}+1} \pi_{s_{1}}^{(p)}$.

Теперь допустим, что утверждение доказано для $k-1$. Тогда

$$
e_{j}^{p} e_{i}^{q}=\sum_{s_{1}, \ldots, s_{k-1}=1}^{c} \lambda_{s_{1} \cdots s_{k-1}} v_{s_{1} \cdots s_{k-1}}+\sum_{v_{t}^{(k-1)} \in \mathcal{S}_{i j}} \lambda_{t}^{(k-1)} v_{t}^{(k-1)} .
$$


Рассмотрим моном $v_{s_{1} \cdots s_{k-1}}=e_{j}^{p-\sum_{m=1}^{k-1} s_{m}} T_{j}^{s_{k-1}}\left(e_{i}\right) \cdots T_{j}^{s_{1}}\left(e_{i}\right) e_{i}^{q-k+1}$. Пользуясь соотношением (3.2), имеем

$$
\begin{aligned}
v_{s_{1} \cdots s_{k-1}}= & e_{j}^{p-\sum_{m=1}^{k-1} s_{m}} e_{i} T_{j}^{s_{k-1}}\left(e_{i}\right) \cdots T_{j}^{s_{1}}\left(e_{i}\right) e_{i}^{q-k} \\
& -e_{j}^{p-\sum_{m=1}^{k-1} s_{m}} T_{i}\left(T_{j}^{s_{k-1}}\left(e_{i}\right) \cdots T_{j}^{s_{1}}\left(e_{i}\right)\right) e_{i}^{q-k} \\
= & T_{i}\left(e_{j}^{p-\sum_{m=1}^{k-1} s_{m}}\right) T_{j}^{s_{k-1}}\left(e_{i}\right) \cdots T_{j}^{s_{1}}\left(e_{i}\right) e_{i}^{q-k} \\
& +e_{i} e_{j}^{p-\sum_{m=1}^{k-1} s_{m}} T_{j}^{s_{k-1}}\left(e_{i}\right) \cdots T_{j}^{s_{1}}\left(e_{i}\right) e_{i}^{q-k} \\
& -\sum_{m=1}^{k-1} e_{j}^{p-\sum_{m=1}^{k-1} s_{m}} T_{j}^{s_{k-1}}\left(e_{i}\right) \cdots T_{i} T_{j}^{s_{m}}\left(e_{i}\right) \cdots T_{j}^{s_{1}}\left(e_{i}\right) e_{i}^{q-k} \\
= & \sum_{s_{k}=}^{c}(-1)^{s_{k}+1} \pi_{s_{k}}^{\left(p-\sum_{m=1}^{k-1} s_{m}\right)} e_{j}^{p-\sum_{m=1}^{k} s_{m}} T_{j}^{s_{k}}\left(e_{i}\right) T_{j}^{s_{k-1}}\left(e_{i}\right) \cdots T_{j}^{s_{1}}\left(e_{i}\right) e_{i}^{q-k} \\
& -\sum_{m=1}^{k-1} e_{j}^{p-\sum_{m=1}^{k-1} s_{m}} T_{j}^{s_{k-1}}\left(e_{i}\right) \cdots T_{i} T_{j}^{s_{m}}\left(e_{i}\right) \cdots T_{j}^{s_{1}}\left(e_{i}\right) e_{i}^{q-k} \\
& +e_{i} e_{j}^{p-\sum_{m=1}^{k-1} s_{m}} T_{j}^{s_{k-1}}\left(e_{i}\right) \cdots T_{j}^{s_{1}}\left(e_{i}\right) e_{i}^{q-k} .
\end{aligned}
$$

Отсюда вытекает, что

$$
\begin{aligned}
e_{j}^{p} e_{i}^{q}= & \sum_{s_{1}, \ldots, s_{k-1}=1}^{c} \sum_{s_{k}=1}^{c}(-1)^{s_{k}+1} \lambda_{s_{1} \cdots s_{k-1}} \pi_{s_{k}}^{\left(p-\sum_{m=1}^{k-1} s_{m}\right)} v_{s_{1} \cdots s_{k}} \\
& -\sum_{s_{1}, \ldots, s_{k-1}=1}^{c} \sum_{m=1}^{k-1} \lambda_{s_{1} \cdots s_{k-1}} e_{j}^{p-\sum_{m=1}^{k-1} s_{m}} T_{j}^{s_{k-1}}\left(e_{i}\right) \cdots T_{i} T_{j}^{s_{m}}\left(e_{i}\right) \cdots T_{j}^{s_{1}}\left(e_{i}\right) e_{i}^{q-k} \\
& +\sum_{s_{1}, \ldots, s_{k-1}=1}^{c} \lambda_{s_{1} \cdots s_{k-1}} e_{i} e_{j}^{p-\sum_{m=1}^{k-1} s_{m}} T_{j}^{s_{k-1}}\left(e_{i}\right) \cdots T_{j}^{s_{1}}\left(e_{i}\right) e_{i}^{q-k} \\
& +\sum_{v_{t}^{(k-1)} \in \mathcal{S}_{i j}} \lambda_{t}^{(k-1)} v_{t}^{(k-1)} \\
= & \sum_{s_{1}, \ldots, s_{k}=1}^{c} \lambda_{s_{1} \cdots s_{k}} v_{s_{1} \cdots s_{k}}+\sum_{v_{t}^{(k)} \in \mathcal{S}_{i j}} \lambda_{t}^{(k)} v_{t}^{(k)} .
\end{aligned}
$$

Этим доказано требуемое утверждение о разложении.

Скажем, что верхние мономы в последнем разложении $e_{j}^{p} e_{i}^{q}$ являются результатом элементарного преобразования, подчиненного $e_{j}^{p} e_{i}^{q}$.

Пусть теперь $v$ - моном в $\mathcal{U}(\mathfrak{g})$ от элементов базиса $e$, и пусть $\operatorname{deg}(v) \leqslant l$ (в противном случае $\left.\alpha_{I}(v)=0\right)$. Моном $v$ разлагается единственным образом по упорядоченным мономам $e^{J}$. Это разложение получается действием последовательности элементарных преобразований, подчиненных $v$. Заметим, что каждое элементарное преобразование, подчиненное моному $e_{j}^{p} e_{i}^{q}$, не уменьшает его нильпотентную степень. А именно, учитывая, что

$$
T_{j}^{s_{m}}\left(e_{i}\right)=\sum_{b\left(s_{m}\right)>b\left(s_{m}-1\right)>\cdots>b(1)>j} \Gamma_{i j}^{b\left(s_{m}\right)} e_{b\left(s_{m}\right)}
$$


при $\operatorname{deg}\left(e_{b\left(s_{m}\right)}\right) \geqslant \operatorname{deg}\left(e_{i}\right)+s_{m} \operatorname{deg}\left(e_{j}\right), s_{m} \leqslant c^{*}$ и $\Gamma_{i j}^{b\left(s_{m}\right)}=C_{j i}^{b(1)} C_{j b(1)}^{b(2)} \cdots C_{j b\left(s_{m}-1\right)}^{b\left(s_{m}\right)}$, мы получаем

$$
\begin{aligned}
e_{j}^{p} e_{i}^{q}= & \sum_{s_{1}, \ldots, s_{k}=1}^{c} \sum_{i, j} \Gamma_{i j}^{b\left(s_{1}\right)} \cdots \Gamma_{i j}^{b\left(s_{k}\right)} \lambda_{s_{1} \cdots s_{k}} e_{j}^{p-\sum_{m=1}^{k} s_{m}} e_{b\left(s_{k}\right)} \cdots e_{b\left(s_{1}\right)} e_{i}^{q-k} \\
& +\sum_{v_{t}^{(k)} \in \mathcal{S}_{i j}} \lambda_{t}^{(k)} v_{t}^{(k)}, \\
\left|\lambda_{s_{1} \cdots s_{k}}\right|= & \pi_{s_{1}}^{(p)} \pi_{s_{2}}^{\left(p-s_{1}\right)} \cdots \pi_{s_{k}}^{\left(p-\sum_{m=1}^{k-1} s_{m}\right)} \leqslant\left(\begin{array}{c}
p \\
s_{1}
\end{array}\right)\left(\begin{array}{c}
p-s_{1} \\
s_{2}
\end{array}\right) \cdots\left(\begin{array}{c}
p-\sum_{m=1}^{k-1} s_{m} \\
s_{k}
\end{array}\right) \\
\leqslant & p !\left(\left(p-\sum_{m=1}^{k} s_{m}\right) !\right)^{-1} .
\end{aligned}
$$

Отсюда вытекает, что

$$
\left|\sum_{i, j} \Gamma_{i j}^{b\left(s_{1}\right)} \cdots \Gamma_{i j}^{b\left(s_{k}\right)} \lambda_{s_{1} \cdots s_{k}}\right| \leqslant\left(\left(n-d_{1}\right) C\right)^{\beta} p !((p-\beta) !)^{-1}
$$

где $\beta=\sum_{m=1}^{k} s_{m}$.

Пусть теперь $e_{j}^{p_{j}^{(\tau)}} e_{i}^{p_{i}^{(\sigma)}}, d_{\sigma-1}<i \leqslant d_{\sigma}, d_{\tau-1}<j \leqslant d_{\tau}, \sigma \leqslant \tau,-$ это все мономы, участвующие в последовательных элементарных преобразованиях, подчиненных $v$, с помощью которых получается базисный моном $e^{I}$. Тогда коэффициенты всех мономов, полученных этими элементарными преобразованиями, по модулю больше, чем

$$
\left(c\left(n-d_{1}\right) C\right)^{\sum_{i, j} \beta_{i j}^{(\sigma, \tau)}} \prod_{\sigma \leqslant \tau, i, j} p_{j}^{(\tau)} !\left(\left(p_{j}^{(\tau)}-\beta_{i j}^{(\sigma, \tau)}\right) !\right)^{-1}, \quad \beta_{i j}^{(\sigma, \tau)}=\sum_{m=1}^{k_{i j}^{(\sigma, \tau)}} s_{m}^{(\sigma, \tau)}
$$

Упростим последнее произведение в коэффициенте при базисном мономе $e^{I}$. При фиксированных $\tau$ и $\sigma, \sigma \leqslant \tau$, обозначим через $\Lambda_{\sigma, \tau}$ множество всех индексов $j$ таких, что моном $e_{j}^{p_{j}^{(\tau)}} e_{i}^{p_{i}^{(\sigma)}}$ появляется в процедуре элементарных преобразований для некоторого $i, d_{\sigma-1}<i \leqslant d_{\sigma}$. Множество всех таких индексов $i$ для данного $j$ обозначается через $M_{j \sigma}$. Заметим, что (см. (3.3))

$$
\prod_{\sigma \leqslant \tau} \prod_{i, j} p_{j}^{(\tau)} !\left(\left(p_{j}^{(\tau)}-\beta_{i j}^{(\sigma, \tau)}\right) !\right)^{-1}=\prod_{\sigma \leqslant \tau} \prod_{j \in \Lambda_{\sigma, \tau}} p_{j}^{(\tau)} !\left(\left(p_{j}^{(\tau)}-\beta_{j}^{(\tau)}\right) !\right)^{-1}
$$

где $\beta_{j}^{(\tau)}=\sum_{i \in M_{j}} \beta_{i j}^{(\sigma, \tau)}, M_{j}=\bigcup_{\sigma \leqslant \tau} M_{j \sigma}$. Далее,

$$
\prod_{\sigma \leqslant \tau} \prod_{j \in \Lambda_{\sigma, \tau}} p_{j}^{(\tau)} !\left(\left(p_{j}^{(\tau)}-\beta_{j}^{(\tau)}\right) !\right)^{-1} \leqslant \frac{P_{\tau} !}{\left(P_{\tau}-\beta_{\tau}\right) !}
$$


где $P_{\tau}=\sum_{\sigma \leqslant \tau} \sum_{j \in \Lambda_{\sigma, \tau}} p_{j}^{(\tau)}, \beta_{\tau}=\sum_{\sigma \leqslant \tau} \sum_{j \in \Lambda_{\sigma, \tau}} \beta_{j}^{(\tau)}$. Из равенства (3.3) вытекает, что $P_{\tau}-\beta_{\tau} \leqslant\left|I_{\tau}\right|$. Кроме того,

$$
\begin{aligned}
\beta_{\tau} & =\sum_{\sigma \leqslant \tau} \sum_{j \in \Lambda_{\sigma, \tau}, i \in M_{j \sigma}} \sum_{m=1}^{k_{i j}^{(\sigma, \tau)}} s_{m}^{(\sigma, \tau)} \\
& \leqslant \sum_{\sigma \leqslant \tau} \sum_{j \in \Lambda_{\sigma, \tau}, i \in M_{j \sigma}} \sum_{m=1}^{k_{i j}^{(\sigma, \tau)}} c^{*} \wedge \frac{\operatorname{deg}\left(e_{b\left(s_{m}^{(\sigma, \tau)}\right)}\right)-\operatorname{deg}\left(e_{i}\right)}{\operatorname{deg}\left(e_{j}\right)} \\
& =\sum_{\sigma \leqslant \tau} \sum_{j \in \Lambda_{\sigma, \tau}, i \in M_{j \sigma}} \sum_{m=1}^{k_{i j}^{(\sigma, \tau)}} c^{*} \wedge \frac{\operatorname{deg}\left(e_{b\left(s_{m}^{(\sigma, \tau)}\right)}\right)-\sigma}{\tau} \\
& \leqslant \sum_{\sigma \leqslant \tau} \sum_{j \in \Lambda_{\sigma, \tau}, i \in M_{j \sigma}} \sum_{m=1}^{k_{i j}^{(\sigma, \tau)}} c^{*} \wedge \frac{\operatorname{deg}\left(e_{b\left(s_{m}^{(\sigma, \tau)}\right)}\right)-1}{\tau} .
\end{aligned}
$$

Докажем теперь, что

$$
\sum_{\sigma \leqslant \tau} \sum_{j \in \Lambda_{\sigma, \tau}, i \in M_{j \sigma}} \sum_{m=1}^{k_{i j}^{(\sigma, \tau)}} c^{*} \wedge \frac{\operatorname{deg}\left(e_{b\left(s_{m}^{(\sigma, \tau)}\right)}\right)-1}{\tau} \leqslant \sum_{\kappa=\tau+1}^{c} c^{*} \wedge(\kappa-\tau)\left|I_{\kappa}\right|
$$

для всех $\tau, 1 \leqslant \tau \leqslant c-1$. Применим индукцию по $\tau=c-k, 1 \leqslant k \leqslant c-1$.

При $\tau=c-1$, пользуясь (3.3), получаем

$$
\begin{gathered}
\sum_{\sigma \leqslant c-1} \sum_{j \in \Lambda_{\sigma, c-1}, i \in M_{j \sigma}} \sum_{m=1}^{k_{i j}^{(\sigma, c-1)}} c^{*} \wedge \frac{\operatorname{deg}\left(e_{b\left(s_{m}^{(\sigma, c-1)}\right)}\right)-1}{c-1} \\
=\sum_{j \in \Lambda_{1, c-1}, i \in M_{j \sigma}} \sum_{m=1}^{k_{i j}^{(1, c-1)}} c^{*} \wedge 1 \leqslant\left|I_{c}\right| .
\end{gathered}
$$

Пусть $\tau<c-1$. Тогда

$$
\sum_{\sigma \leqslant \tau} \sum_{j \in \Lambda_{\sigma, \tau}, i \in M_{j \sigma}} \sum_{m=1}^{k_{i j}^{(\sigma, \tau)}} c^{*} \wedge \frac{\operatorname{deg}\left(e_{b\left(s_{m}^{(\sigma, \tau)}\right)}\right)-1}{\tau} \leqslant \sum_{\kappa=\tau+1}^{c} c^{*} \wedge(\kappa-\tau) N_{\kappa}^{(\tau)}
$$

где $N_{\kappa}^{(\tau)}$ - число всех $e_{b\left(s_{m}^{(\sigma, \tau)}\right)}, \operatorname{deg}\left(e_{b\left(s_{m}^{(\sigma, \tau)}\right)}\right)=\kappa$. Если $N_{\kappa}^{(\tau)} \leqslant\left|I_{\kappa}\right|$ для всех $\kappa$, $\kappa>\tau$, то доказательство очевидно. Пусть $N_{\lambda}^{(\tau)}>\left|I_{\lambda}\right|$ при некотором $\lambda \geqslant \tau+1$ и $N_{\kappa}^{(\tau)} \leqslant\left|I_{\kappa}\right|$ для всех $\kappa, \tau+1 \leqslant \kappa<\lambda$. Это означает, что некоторые элементы $e_{b\left(s_{m}^{(\sigma, \tau)}\right)}, \operatorname{deg}\left(e_{b\left(s_{m}^{(\sigma, \tau)}\right)}\right)=\lambda$, должны возникать при других элементарных преобразованиях. Иначе мы не могли бы получить базисный моном $e^{I}$. По 
предположению индукции получаем

$$
\begin{aligned}
N_{\lambda}^{(\tau)} & =\sum_{\sigma \leqslant \lambda} \sum_{j \in \Lambda_{\sigma, \lambda}^{*}} p_{j}^{(\lambda)}=\sum_{\sigma \leqslant \lambda} \sum_{j \in \Lambda_{\sigma, \lambda}^{*}}\left(p_{j}^{(\lambda)}-\beta_{j}^{(\lambda)}\right)+\sum_{\sigma \leqslant \lambda} \sum_{j \in \Lambda_{\sigma, \lambda}^{*}} \beta_{j}^{(\lambda)} \\
& \leqslant\left|I_{\lambda}\right|+\sum_{\alpha=\lambda+1}^{c} c^{*} \wedge(\alpha-\lambda) N_{\alpha}^{(\lambda)}
\end{aligned}
$$

где $\Lambda_{\sigma, \lambda}^{*}$ - соответствующее подмножество $\Lambda_{\sigma, \lambda}$, а $N_{\alpha}^{(\lambda)}$ - число некоторых элементов базиса $e$, имеющих нильпотентную степень $\alpha$. Отсюда следует, что

$$
\sum_{\kappa=\tau+1}^{c} c^{*} \wedge(\kappa-\tau) N_{\kappa}^{(\tau)} \leqslant \sum_{\kappa=\tau+1}^{\lambda} c^{*} \wedge(\kappa-\tau)\left|I_{\kappa}\right|+\sum_{\kappa=\lambda+1}^{c} c^{*} \wedge(\kappa-\tau)\left(N_{\kappa}^{(\lambda)}+N_{\kappa}^{(\tau)}\right) .
$$

Используя такое же рассуждение для $N_{\kappa}^{(\lambda)}+N_{\kappa}^{(\tau)}, \kappa>\lambda$, получим

$$
\sum_{\kappa=\tau+1}^{c} c^{*} \wedge(\kappa-\tau) N_{\kappa}^{(\tau)} \leqslant \sum_{\kappa=\tau+1}^{c} c^{*} \wedge(\kappa-\tau)\left|I_{\kappa}\right|
$$

Тем самым неравенство (3.4) доказано.

Теперь, пользуясь (3.4), мы выводим, что

$$
\begin{aligned}
\prod_{\sigma \leqslant \tau, i, j} p_{j}^{(\tau)} !\left(\left(p_{j}^{(\tau)}-\beta_{i j}^{(\sigma, \tau)}\right) !\right)^{-1} & =\prod_{\tau=1}^{c-1} \prod_{\sigma \leqslant \tau} \prod_{j \in \Lambda_{\sigma, \tau}} p_{j}^{(\tau)} !\left(\left(p_{j}^{(\tau)}-\beta_{j}^{(\tau)}\right) !\right)^{-1} \\
& \leqslant \prod_{\tau=1}^{c-1} \frac{\left(P_{\tau}-\beta_{\tau}+\beta_{\tau}\right) !}{\left(P_{\tau}-\beta_{\tau}\right) !} \leqslant \prod_{\tau=1}^{c-1} \frac{\left(\left|I_{\tau}\right|+\beta_{\tau}\right) !}{\left|I_{\tau}\right| !} \\
& \leqslant \prod_{\tau=1}^{c-1} \frac{\left(\left|I_{\tau}\right|+\sum_{\kappa=\tau+1}^{c} c^{*} \wedge(\kappa-\tau)\left|I_{\kappa}\right|\right) !}{\left|I_{\tau}\right| !}
\end{aligned}
$$

Для завершения доказательства применим следующую оценку:

$$
\begin{aligned}
\sum_{i, j} \beta_{i j}^{(\sigma, \tau)} & =\sum_{\tau=1}^{c-1} \beta_{\tau} \leqslant \sum_{\tau=1}^{c-1} \sum_{\kappa=\tau+1}^{c} c^{*} \wedge(\kappa-\tau)\left|I_{\kappa}\right| \\
& \leqslant \sum_{\tau=2}^{c}\left(\sum_{m=1}^{\tau-1} c^{*} \wedge m\right)\left|I_{\tau}\right| \leqslant \sum_{\tau=2}^{c}\left(\sum_{m=1}^{c-1} c^{*} \wedge m\right)\left|I_{\tau}\right| \leqslant c^{2} \sum_{\tau=2}^{c}\left|I_{\tau}\right| \leqslant c^{2}|I| .
\end{aligned}
$$

Тогда

$$
\left(c\left(n-d_{1}\right) C\right)^{\sum_{i, j} \beta_{i j}^{(\sigma, \tau)}} \prod_{\tau, \sigma \leqslant \tau, i, j} p_{j}^{(\tau)} !\left(\left(p_{j}^{(\tau)}-\beta_{i j}^{(\sigma, \tau)}\right) !\right)^{-1} \leqslant \theta_{I},
$$

откуда получаем $\left|\alpha_{I}(v)\right| \leqslant \theta_{I}$. Лемма доказана.

ЗАмечАниЕ 3.1. Оценку из леммы 3.2 можно упростить в случае наличия в $\mathfrak{g}$ специального треугольного базиса $e$. Например, допустим, что $\left[\left[e_{i}, e_{j}\right], e_{j}\right]=0$ для всех $i, j, i<j$. Тогда $c^{*}=1$ и

$$
\left\|\alpha_{I}\right\|_{\mathcal{A}\left(l_{e}\right)^{*}} \leqslant \varkappa^{c^{2}|I|} \prod_{\tau=1}^{c-1} \frac{\left(\left|I_{\tau}\right|+\cdots+\left|I_{c}\right|\right) !}{\left|I_{\tau}\right| !}
$$

Такие базисы называются базисами Кляйнеке-Широкова (см. ниже п. 3.2). Они имеют ряд преимуществ, обсуждаемых в следующем пункте. 
3.2. Рост треугольного базиса. Пусть $\mathfrak{g}$ - нильпотентная алгебра Ли, $c$ - ее степень нильпотентности, $e=\left(e_{1}, \ldots, e_{n}\right)=\mathfrak{e}_{1} \cup \cdots \cup \mathfrak{e}_{c}-$ ее треугольный базис, $\mathfrak{e}_{s}=\left(e_{d_{s-1}+1}, \ldots, e_{d_{s}}\right) \in \mathfrak{g}^{(s)} \backslash \mathfrak{g}^{(s+1)}, c^{*}=\max \left\{s: \operatorname{ad}^{s}\left(e_{j}\right)\left(e_{i}\right) \neq 0, i<j\right\}$, и пусть $\mathcal{A}$ - банахова алгебра, обертывающая $\mathfrak{g}$, т. е. $\mathfrak{g}$ вложена в банахову алгебру $\mathcal{A}$ таким образом, что ассоциативная подалгебра в $\mathcal{A}$, порожденная алгеброй $\mathfrak{g}$, плотна в $\mathcal{A}$. Согласно [14], [15] алгебра $\mathcal{A}$ коммутативна по модулю ее радикала Джекобсона. Поэтому $[\mathfrak{g}, \mathfrak{g}] \subseteq \operatorname{Rad} \mathcal{A}$ и все $e_{i}, i>d_{1}$, квазинильпотентны в $\mathcal{A}$.

Обозначим через $\mathbb{R}_{+}^{0}\left[x_{1}, \ldots, x_{d_{1}}\right]$ конус всех полиномов от $d_{1}$ коммутирующих переменных $x=\left(x_{1}, \ldots, x_{d_{1}}\right)$ с положительными коэффициентами и с нулевым свободным членом. Для любой банаховой алгебры, обертывающей $\mathfrak{g}$, будем через $\left\|\mathfrak{e}_{1}\right\|_{\mathcal{A}}$ обозначать набор $\left(\left\|e_{1}\right\|_{\mathcal{A}}, \ldots,\left\|e_{d_{1}}\right\|_{\mathcal{A}}\right)$.

ОПРеДЕЛЕНИЕ 3.1. Пусть $\omega=\left\{\omega_{k}^{(i)} \in \mathbb{R}_{+}: d_{1}<i \leqslant n, k \in \mathbb{N}\right\}$ - такая последовательность, что $\lim _{k} \omega_{k}^{(i)}=0$ для всех $i$. Скажем, что треугольный базис $e$ алгебры $\mathfrak{g}$ имеет рост $\omega$, если существуют полиномы $\gamma_{i}(x) \in \mathbb{R}_{+}^{0}\left[x_{1}, \ldots, x_{d_{1}}\right]$ такие, что $\left\|e_{i}^{k}\right\|_{\mathcal{A}}^{1 / k} \leqslant \gamma_{i}\left(\left\|\mathfrak{e}_{1}\right\|_{\mathcal{A}}\right) \omega_{k}^{(i)}$ для каждой банаховой алгебры $\mathcal{A}$, обертывающей $\mathfrak{g}, i>d_{1}$. В частности, $\left\|e_{i}^{k}\right\|_{\mathcal{A}}^{1 / k}=O\left(\omega_{k}^{(i)}\right), i>d_{1}$. Скажем также, что $e$ имеет нормальный рост, если $\omega_{k}^{(i)}=(1 / k)^{\theta_{i}}$ для всех $i$, где $\theta_{i} \geqslant \sum_{m=1}^{\tau-1} c^{*} \wedge m$ для всех $e_{i} \in \mathfrak{e}_{\tau}$.

Появление полиномов $\gamma_{i}(x)$ из конуса иллюстрируется следующими примерами.

ПримеР 3.1. Пусть $\mathfrak{g}$ - нильпотентная алгебра Ли степени нильпотентности $2, \mathcal{A}$ - банахова алгебра, обертывающая $\mathfrak{g}$, а $e$ - треугольный базис в $\mathfrak{g}$, ассоциированный с образующими Ли $e_{1}, \ldots, e_{d_{1}}$ (см. п. 3.1). Фиксируем $i$, $i>d_{1}$. Тогда $e_{i}=\left[e_{s}, e_{t}\right]$ для некоторых $s, t, 1 \leqslant s<t \leqslant d_{1}$. Учитывая, что $\mathfrak{g}^{(3)}=\{0\}$, мы выводим формулу Кляйнеке-Широкова $k ! e_{i}^{k}=T_{s}^{k}\left(e_{t}^{k}\right), k \in \mathbb{N}$, где $T_{s} \in \mathcal{L}(\mathcal{A}), T_{s}=L_{e_{s}}-R_{e_{s}}$. Тогда

$$
\left\|e_{i}^{k}\right\|_{\mathcal{A}} \leqslant(k !)^{-1}\left\|T_{s}\right\|_{\mathcal{A}}^{k}\left\|e_{t}\right\|_{\mathcal{A}}^{k} \leqslant(k !)^{-1}\left(2\left\|e_{s}\right\|_{\mathcal{A}}\left\|e_{t}\right\|_{\mathcal{A}}\right)^{k},
$$

где $\left\|T_{s}\right\|_{\mathcal{A}}$ - операторная норма $T_{s}$. Отсюда $\left\|e_{i}^{k}\right\|_{\mathcal{A}}^{1 / k} \leqslant(k !)^{-1 / k} \gamma_{i}\left(\left\|\mathfrak{e}_{1}\right\|_{\mathcal{A}}\right)$, где $\gamma_{i}(x)=2 x_{s} x_{t}-$ моном из конуса $\mathbb{R}_{+}^{0}\left[x_{1}, \ldots, x_{d_{1}}\right]$. Но по формуле Стирлинга имеем $(k !)^{-1 / k} \sim k^{-1}$ с точностью до константы. Тем самым, $\left\|e_{i}^{k}\right\|_{\mathcal{A}}^{1 / k}=O\left(k^{-1}\right)$. Таким образом, рост базиса е задается последовательностью $\omega=\left\{\omega_{k}^{(i)}\right\}, \omega_{k}^{(i)}=k^{-1}$ для всех $i, i>d_{1}, k \in \mathbb{N}$, т. е. базис $e$ имеет нормальный рост.

Следующее предложение доставляет более сложный пример.

ПРеДЛОжЕНИЕ 3.2. Пустъ $\mathfrak{g}$ - нильпотентная алгебра Ли степени нильпотентности с, а е-ее треугольный базис, ассоциированный с образующими Ли $e_{1}, \ldots, e_{d_{1}}$. Положим $\omega_{k}^{(i)}=(1 / k)^{\tau-1} n$ ри $d_{\tau-1}<i \leqslant d_{\tau}, 2 \leqslant \tau \leqslant c$, $k \in \mathbb{N}$. Если $\left[\left[e_{i}, e_{j}\right], e_{j}\right]=0$ для всех $i<j$ (в этом случае $\left.c^{*}=1\right), \operatorname{mo} \omega=\left\{\omega_{k}^{(i)}\right\}$ есть рост базиса е.

ДокаЗАТЕЛЬство. Фиксируем $i, i>d_{1}$, и будем считать, что $d_{\tau-1}<i \leqslant d_{\tau}$ (тем самым, $\operatorname{deg}\left(e_{i}\right)=\tau$ ). Покажем сначала, что $e_{i}=\left[e_{s}, e_{t}\right]$ для некоторых $s$, 
$1 \leqslant s \leqslant d_{1}$, и $t, d_{\tau-2}<t \leqslant d_{\tau-1}$. При $\tau=2$ это очевидно. Пусть $\tau>2$. По определению (см. п. 3.1) $e_{i}$ есть лиево слово длины $\tau$ от образующих Ли $e_{1}, \ldots, e_{d_{1}}$. Тогда $e_{i}=\left[e_{s}, u\right]$ для некоторого $s, 1 \leqslant s \leqslant d_{1}$, и некоторого лиева слова $u$ длины $\tau-1$. Однако $u=\sum_{d_{\tau-2}<t \leqslant d_{\tau-1}} a_{t} e_{t}+\sum_{t>d_{\tau-1}} a_{t} e_{t}$, откуда

$$
e_{i}=\sum_{d_{\tau-2}<t \leqslant d_{\tau-1}} a_{t}\left[e_{s}, e_{t}\right]+\sum_{t>d_{\tau-1}} a_{t}\left[e_{s}, e_{t}\right] \in \sum_{\operatorname{deg}\left(\left[e_{s}, e_{t}\right]\right)=\tau} a_{t}\left[e_{s}, e_{t}\right]+\mathfrak{g}^{(\tau+1)} .
$$

Пусть $\left(\left[e_{s}, e_{t_{1}^{*}}\right], \ldots,\left[e_{s}, e_{t_{m}^{*}}\right]\right)-$ свободная часть множества коммутаторов

$$
\left\{\left[e_{s}, e_{t}\right]: 1 \leqslant s \leqslant d_{1}, d_{\tau-2}<t \leqslant d_{\tau-1}, \operatorname{deg}\left(\left[e_{s}, e_{t}\right]\right)=\tau\right\} .
$$

Последние элементы принадлежат базису $e$. Кроме того, $e_{i}-\sum_{k=1}^{m} b_{k}\left[e_{s}, e_{t_{k}^{*}}\right] \in$ $\mathfrak{g}^{(\tau+1)}$ для некоторого $b_{k}$. Поэтому $e_{i}-\sum_{k=1}^{m} b_{k}\left[e_{s}, e_{t_{k}^{*}}\right]=\sum_{j>d_{\tau}} \alpha_{j} e_{j}$. Однако $e_{i}$ также был элементом базиса $e$, так что $e_{i}-\sum_{k=1}^{m} b_{k}\left[e_{s}, e_{t_{k}^{*}}\right]=0$ и $e_{i}=\left[e_{s}, e_{t_{k}^{*}}\right]$ для некоторого $k$.

Пусть теперь $\mathcal{A}$ - банахова алгебра, обертывающая алгебру Ли $\mathfrak{g}$. Докажем предложение индукцией по $\tau$. Как в примере 3.1 , получаем, что $(-1)^{k} k ! e_{i}^{k}=$ $T_{t}^{k}\left(e_{s}^{k}\right)$ и $\left\|e_{i}^{k}\right\|_{\mathcal{A}} \leqslant(k !)^{-1}\left\|e_{s}\right\|_{\mathcal{A}}^{k}\left\|T_{t}^{k}\right\|_{\mathcal{A}}$, где $T_{t} \in \mathcal{L}(\mathcal{A}), T_{t}=L_{e_{t}}-R_{e_{t}}$. В частности, для $\tau=2$ имеем

$$
\left\|e_{i}^{k}\right\|_{\mathcal{A}} \leqslant(k !)^{-1}\left(2\left\|e_{s}\right\|_{\mathcal{A}}\left\|e_{t}\right\|_{\mathcal{A}}\right)^{k} .
$$

Следовательно, $\left\|e_{i}^{k}\right\|_{\mathcal{A}}^{1 / k} \leqslant k^{-1} \gamma_{i}\left(\left\|\mathfrak{e}_{1}\right\|_{\mathcal{A}}\right)$, где $\gamma_{i}(x)=2 x_{s} x_{t}$.

При $\tau>2$ имеем $\operatorname{deg}\left(e_{t}\right)=\tau-1$ и по предположению индукции получаем $\left\|e_{t}^{k}\right\|_{\mathcal{A}} \leqslant \gamma_{t}\left(\left\|\mathfrak{e}_{1}\right\|_{\mathcal{A}}\right)^{k}(1 / k !)^{\tau-2}$ для некоторого $\gamma_{t}(x) \in \mathbb{R}_{+}^{0}\left[x_{1}, \ldots, x_{d_{1}}\right]$. Тогда

$$
\begin{aligned}
\left\|T_{t}^{k}\right\|_{\mathcal{A}} & =\left\|\sum_{m=0}^{k}\left(\begin{array}{c}
k \\
m
\end{array}\right) L_{e_{t}}^{k-m} R_{e_{t}}^{m}\right\|_{\mathcal{A}} \leqslant \sum_{m=0}^{k}\left(\begin{array}{c}
k \\
m
\end{array}\right)\left\|e_{t}^{k-m}\right\|_{\mathcal{A}}\left\|_{e_{t}^{m}}\right\|_{\mathcal{A}} \\
& \leqslant \gamma_{t}\left(\left\|\mathfrak{e}_{1}\right\|_{\mathcal{A}}\right)^{k} \sum_{m=0}^{k}\left(\begin{array}{c}
k \\
m
\end{array}\right)\left(\frac{1}{(k-m) ! m !}\right)^{\tau-2} \\
& \leqslant \gamma_{t}\left(\left\|\mathfrak{e}_{1}\right\|_{\mathcal{A}}\right)^{k}\left(\frac{1}{k !}\right)^{\tau-2} \sum_{m=0}^{k}\left(\begin{array}{c}
k \\
m
\end{array}\right)^{\tau-1} \leqslant\left(2^{\tau-1} \gamma_{t}\left(\left\|\mathfrak{e}_{1}\right\|_{\mathcal{A}}\right)\right)^{k}\left(\frac{1}{k !}\right)^{\tau-2} .
\end{aligned}
$$

Отсюда вытекает, что $\left\|e_{i}^{k}\right\|_{\mathcal{A}} \leqslant \gamma_{i}\left(\left\|\mathfrak{e}_{1}\right\|_{\mathcal{A}}\right)^{k}(1 / k !)^{\tau-1}$, где $\gamma_{i}(x)=2^{\tau-1} x_{s} \gamma_{t}(x) \in$ $\mathbb{R}_{+}^{0}\left[x_{1}, \ldots, x_{d_{1}}\right]$. Предложение доказано.

Треугольный базис, возникающий в предложении 3.2, называется базисом Кляйнеке-Широкова. Таким образом, рост базиса Кляйнеке-Широкова нормален. Отметим в заключение, что примером базиса Кляйнеке-Широкова является канонический базис алгебры Ли всех строго верхнетреугольных матриц.

\section{§ 4. Голоморфные функции}

В этом параграфе изучается "задача об абсолютном базисе” в алгебре всех голоморфных (в частности, целых) функций от элементов нильпотентной алгебры Ли $\mathfrak{g}$. 
4.1. Алгебры Фреше $\mathcal{O}_{\mathfrak{g}}\left(D_{r}\right)$. Пусть $F$ - конечномерная алгебра Ли, а $e=\left(e_{1}, \ldots, e_{n}\right)$ - такой ее базис, что $\left(e_{m+1}, \ldots, e_{n}\right)$ является базисом в $[F, F]$ для некоторого $m$. Пространство $\Delta(F)$ всех характеров Ли отождествляется с пространством $\mathbb{C}^{m}$ посредством отображения $\Delta(F) \rightarrow \mathbb{C}^{m}, a \mapsto\left(a\left(e_{1}\right), \ldots\right.$ $\left.\ldots, a\left(e_{m}\right)\right)$, где $m=\operatorname{dim}\left(F / F^{(2)}\right)$. Рассмотрим в $\mathbb{C}^{n}$ полидиск $D_{r}$ полирадиуса $r=\left(r_{1}, \ldots, r_{n}\right) \in \overline{\mathbb{R}}_{+}^{n}$ с центром в начале координат. Алгебра $\mathcal{O}_{F}\left(D_{r}\right)$ всех голоморфных функиий от элементов алгебры Ли $F$ определяется (см. [8], [9]) как хаусдорфово пополнение Фреше универсальной обертывающей алгебры $\mathcal{U}(F)$ относительно топологии, порожденной семейством полунорм $\left\{\|\cdot\|_{\mathcal{A}\left(l_{e, s}\right)}\right.$ : $s<r\}$, где $\|\cdot\|_{\mathcal{A}\left(l_{e, s}\right)}$ - норма на $\mathcal{U}(F)$, наследованная из банаховой обертывающей алгебры $\mathcal{A}\left(l_{e, s}\right)$ (см. $\left.\S 3\right)$ относительно $\ell_{1}$-нормы $l_{e, s}$ на $F$. Если $r=\infty$ (т. е. все $\left.r_{i}=+\infty\right)$, то $\mathcal{O}_{F}\left(\mathbb{C}^{n}\right)$ называется алгеброй всех цельх функций от элементов базиса е. Известно [8], что если $\mathfrak{g}$ - разрешимая алгебра Ли, то пространство всех непрерывных характеров на $\mathcal{O}_{\mathfrak{g}}\left(D_{r}\right)$ отождествляется с суженным полидиском $D_{r_{\mathrm{s}}} \subseteq \mathbb{C}^{m}=\Delta(\mathfrak{g})$, где $r_{\mathrm{s}}=\left(r_{1}, \ldots, r_{m}\right), m=\operatorname{dim}\left(\mathfrak{g} / \mathfrak{g}^{(2)}\right)$. Кроме того, если $r_{\mathrm{s}}=t_{\mathrm{s}}$, то $\mathcal{O}_{\mathfrak{g}}\left(D_{r}\right)=\mathcal{O}_{\mathfrak{g}}\left(D_{t}\right)$ с точностью до топологического изоморфизма алгебр (см. [9]). Поэтому алгебра $\mathcal{O}_{\mathfrak{g}}\left(D_{r}\right)$ не зависит от радикальной части $r_{m+1}, \ldots, r_{n}$, а полностью определяется полупростой частью $r_{\mathrm{s}}$. Доказано также, что факторалгебра $\mathcal{O}_{\mathfrak{g}}\left(D_{r}\right) / \operatorname{Rad} \mathcal{O}_{\mathfrak{g}}\left(D_{r}\right)$ по радикалу Джекобсона топологически изоморфна алгебре $\mathcal{O}\left(D_{r_{\mathrm{s}}}\right)$ всех обычных голоморфных функций на полидиске $D_{r_{\mathrm{s}}} \subseteq \Delta(\mathfrak{g})$.

Заметим, что алгебра $\mathcal{O}_{F}\left(D_{r}\right)$ отождествляется с обратным пределом банаховых обертывающих алгебр. А именно, рассмотрим введенное в $\S 3$ семейство $\mathcal{A}\left(l_{e, s}\right), 0<s<r$, банаховых обертывающих алгебр с каноническими вложениями $\varphi_{s}: F \rightarrow \mathcal{A}\left(l_{e, s}\right)$. Возьмем $s, t, 0<t \leqslant s<r$, и $x=\sum_{i} a_{i}\left(s_{i}^{-1} e_{i}\right) \in F$. Тогда

$$
\left\|\varphi_{t}(x)\right\|_{\mathcal{A}\left(l_{e, t}\right)} \leqslant \max \left(s^{-1} t\right) l_{e, s}(x) \leqslant l_{e, s}(x),
$$

так что $\left\|\varphi_{t}\right\| \leqslant 1$ в предположении, что алгебра $F$ снабжена $\ell_{1}$-нормой $l_{e, s}$ относительно базиса $s^{-1} e$. По лемме 3.1 существует гомоморфизм алгебр $u_{t s}: \mathcal{A}\left(l_{e, s}\right) \rightarrow \mathcal{A}\left(l_{e, t}\right)$ такой, что $u_{t s} \varphi_{s}=\varphi_{t},\left\|u_{t s}\right\| \leqslant 1$. В частности, $u_{t s}$ сводится к тождественному отображению на $\mathcal{U}(F)$. Поэтому $\mathfrak{A}_{e, r}=\left\{\mathcal{A}\left(l_{e, s}\right), u_{t s}\right.$ : $0<s, t<r\}-$ проективная система банаховых алгебр. Пусть $\lim _{\mathfrak{e}, r}-$ ее обратный предел, $\chi_{s}: \lim _{\mathfrak{A}, r} \rightarrow \mathcal{A}\left(l_{e, s}\right), s<r,-$ семейство канонических проекций, а $\varphi_{r}: F \rightarrow \lim \mathfrak{A}_{e, r}, \varphi_{r}(x)=\left(\varphi_{s}(x)\right)_{s<r},-$ гомоморфизм алгебр Ли. Тогда $\lim _{\longleftarrow} \mathfrak{A}_{e, r}$ есть алгебра Аренса-Майкла, являющаяся пополнением $\mathcal{U}(F)$ по Хаусдорфу относительно семейства мультипликативных полунорм $\left\{\|\cdot\|_{\mathcal{A}\left(l_{e, s}\right)}: s<r\right\}$, т. е. $\lim ^{\longleftarrow} \mathfrak{A}_{e, r}=\mathcal{O}_{F}\left(D_{r}\right)$. Для алгебры всех целых функций имеем $\mathcal{O}_{F}\left(\mathbb{C}^{n}\right)=\lim _{e, S} \mathfrak{A}_{e, S}$ где $\subseteq \mathbb{R}_{+}^{n}$ - неограниченно возрастающее подмножество (см. $\S 2)$, а $\mathfrak{A}_{e, S}=\left\{\mathcal{A}\left(l_{e, s}\right), u_{t s}: s, t \in S\right\}$ - сопутствующая проективная система.

Лемма 4.1. Пара $\left(\lim _{\longleftarrow} \mathfrak{A}_{e, S}, \varphi_{S}\right)$ является оболочкой Аренса-Майкла алгебры $\mathcal{U}(F)$, m.е. $\mathcal{O}_{F}=\overleftarrow{\mathcal{O}}_{F}\left(\mathbb{C}^{n}\right)=\lim _{\mathfrak{A}, S}$ с точностъю до топологического изоморфизма для некоторого неограниченно возрастающего подмножества $S \subseteq \mathbb{R}_{+}^{n}$. В частности, $\mathcal{O}_{F}$ есть алгебра Фреше. 
Доказательство. Пусть $\mathcal{A}$ - банахова алгебра, $\pi: \mathcal{U}(F) \rightarrow \mathcal{A}$ - гомоморфизм алгебр, а $k$ - такое натуральное число, что $k \geqslant \max \left\{\left\|\pi\left(e_{i}\right)\right\|_{\mathcal{A}}: 1 \leqslant i \leqslant n\right\}$. Поскольку $S$ - неограниченно возрастающее подмножество $\mathbb{R}_{+}^{n}$, имеем $\min (s) \geqslant$ $k$ для некоторого $s \in S$ (см. §2). Возьмем $x=\sum_{i} a_{i}\left(s_{i}^{-1} e_{i}\right) \in F$. Тогда $\|\pi(x)\|_{\mathcal{A}} \leqslant l_{e, s}(x)(\min (s))^{-1} k \leqslant l_{e, s}(x)$. По лемме 3.1 существует единственный ограниченный гомоморфизм алгебр $\pi^{\prime}: \mathcal{A}\left(l_{e, s}\right) \rightarrow \mathcal{A}$ такой, что $\pi^{\prime} \cdot \varphi_{s}=\pi$. Положим $\tilde{\pi}=\pi^{\prime} \cdot \chi_{s}$, так что $\tilde{\pi}: \lim _{e, S} \rightarrow \mathcal{A}$. Очевидно, $\tilde{\pi} \cdot \varphi_{S}=\pi$. Поскольку образ отображения $\varphi_{S}$ порождает плотную подалгебру в $\lim \mathfrak{A}_{e, S}$, мы заключаем, что гомоморфизм $\widetilde{\pi}$ единствен. Таким образом, пара $\left(\longleftarrow \mathfrak{l i m}_{e, S}, \varphi_{S}\right)$ обладает свойством проективной универсальности (см. п. 2.2). Отсюда вытекает, что $\lim _{\mathfrak{A}_{e, S}}=\mathcal{O}_{F}$ с точностью до топологического изоморфизма. Наконец, заменяя $S$ на счетное неограниченно возрастающее подмножество $\mathbb{R}_{+}^{n}$, мы получаем, что $\mathcal{O}_{F}$ есть алгебра Фреше и Аренса-Майкла. Лемма доказана.

Пусть $\mathfrak{g}$ - конечномерная нильпотентная алгебра Ли с треугольным базисом $e$. Тогда $\mathcal{O}_{\mathfrak{g}}\left(D_{r}\right)$ - окаймленное пополнение $\mathcal{U}(\mathfrak{g})$. Действительно, так как $\|\cdot\|_{\mathcal{A}\left(l_{e, s}\right)}$ - норма на $\mathcal{U}(\mathfrak{g})($ см. $\S 3)$ при каждом $s<r$, то $\mathcal{U}(\mathfrak{g})$ - плотная подалгебра в $\mathcal{O}_{\mathfrak{g}}\left(D_{r}\right)$. Далее, по лемме 3.1 тривиальный характер Ли $\mathfrak{g} \rightarrow \mathbb{C}$ непрерывно продолжается до характера на $\mathcal{O}_{\mathfrak{g}}\left(D_{r}\right)$. Поэтому $\mathcal{O}_{\mathfrak{g}}\left(D_{r}\right)$ есть окаймленное пополнение $\mathcal{U}(\mathfrak{g})$.

Теперь допустим, что $F$ - конечномерная алгебра Ли с базисом $e$. Выберем точку $a \in \Delta(F)$ и рассмотрим подалгебру Ли $F-a$ (см. замечание 2.2$)$ в $\mathcal{U}(F)$, содержащую все элементы $u-a(u), u \in F$. Положим $\mathcal{O}_{F}\left(D_{a, r}\right)=\mathcal{O}_{F-a}\left(D_{r}\right)$ для полидиска $D_{a, r} \subseteq \mathbb{C}^{n}$ полирадиуса $r$ с центром $a$.

ПрЕДЛОЖЕНИЕ 4.1. Пустъ е-треуголъный базис нилъпотентной алгебры Ли $\mathfrak{g}, a \in \Delta(\mathfrak{g})$, а $\mathfrak{S}_{e}\left(D_{a, r}\right)$ - подпространство в $\mathcal{O}_{\mathfrak{g}}\left(D_{a, r}\right)$, состоящее из всех абсолютно сходящихся степеннъх рядов $\sum_{J_{\mathrm{s}}} x_{J_{\mathrm{s}}}\left(e_{\mathrm{s}}-a\right)^{J_{\mathrm{s}}}$. Тогда множество $\mathfrak{m}_{e-a}$ всех упорядоченных мономов $(e-a)^{J}$ от элементов базиса е - а является слабо однородным базисом в $\mathcal{O}_{\mathfrak{g}}\left(D_{a, r}\right)$, который становится однородным, если базис е градуирован. Подпространство $\mathfrak{S}_{e}\left(D_{a, r}\right)$ замкнуто, имеем

$$
\mathcal{O}_{\mathfrak{g}}\left(D_{a, r}\right)=\mathfrak{S}_{e}\left(D_{a, r}\right) \oplus \operatorname{Rad} \mathcal{O}_{\mathfrak{g}}\left(D_{a, r}\right)
$$

и $\mathfrak{S}_{e}\left(D_{a, r}\right)=\mathcal{O}\left(D_{a, r_{\mathrm{s}}}\right)$ с точностью до топологического изоморфизма. Более того, если $\Lambda$ - подмножество в $\mathbb{Z}_{+}^{n}$, a $\mathfrak{S}_{\Lambda}\left(D_{a, r}\right)$ - подпространство в $\mathcal{O}_{\mathfrak{g}}\left(D_{a, r}\right)$, состоящее из всех формальных степенных рядов $\sum_{J} x_{J}(e-a)^{J}$ таких, что $x_{J}=0$ при всех $J \in \Lambda$, mо $\mathfrak{S}_{\Lambda}\left(D_{a, r}\right)$ замкнуто.

ДокАЗАТЕЛЬСтво. Первое утверждение вытекает из предложения 3.1 и определения $\mathcal{O}_{\mathfrak{g}}\left(D_{a, r}\right)$; см. также [9]. Второе утверждение легко сводится к случаю, когда $a=0$, а этот случай был доказан в [9]. Наконец, пусть $\left\{\alpha_{r, J}\right\}-$ семейство координатных линейных функционалов на $\mathcal{O}_{\mathfrak{g}}\left(D_{a, r}\right)$ относительно слабо однородного базиса $\left\{(e-a)^{J}\right\}$ в $\mathcal{O}_{\mathfrak{g}}\left(D_{a, r}\right)$ (см. замечание 2.1). Тогда $f=$

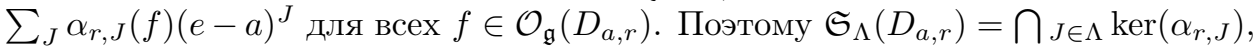
т. е. подпространство $\mathfrak{S}_{\Lambda}\left(D_{a, r}\right)$ замкнуто.

СЛЕДСТвИЕ 4.1. Пусть $\mathfrak{g}$ - нильпотентная алгебра Ли с треугольным базисом $e=\left(e_{1}, \ldots, e_{n}\right)$. Если подмножество $\left\{(e-a)^{J}\right\} \subseteq \mathcal{U}(\mathfrak{g})$ является 
безусловным базисом в $\mathcal{O}_{\mathfrak{g}}\left(D_{a, r}\right)$, то для каждого подмножества $\Lambda \subseteq \mathbb{Z}_{+}^{n}$ имеем разложение $\mathcal{O}_{\mathfrak{g}}\left(D_{a, r}\right)=\mathfrak{S}_{\Lambda}\left(D_{a, r}\right) \oplus \mathfrak{S}_{\mathbb{Z}_{+}^{n} \backslash \Lambda}\left(D_{a, r}\right)$ в топологическую прямую сумму.

ДокАЗАТЕЛЬСтво. Подпространства $\mathfrak{S}_{\Lambda}\left(D_{a, r}\right), \mathfrak{S}_{\mathbb{Z}_{+}^{n} \backslash \Lambda}\left(D_{a, r}\right)$ замкнуты по предложению 4.1. Ясно, что $\mathfrak{S}_{\Lambda}\left(D_{a, r}\right) \cap \mathfrak{S}_{\mathbb{Z}_{+}^{n} \backslash \Lambda}\left(D_{a, r}\right)=\{0\}$ (см. замечание 2.1). Теперь возьмем $f \in \mathcal{O}_{\mathfrak{g}}\left(D_{a, r}\right)$. Тогда $f$ единственным образом разлагается в безусловно сходящийся степенной ряд: $f=\sum_{J} x_{J}(e-a)^{J}$. Следовательно, его подряды $f_{1}=\sum_{J \in \Lambda} x_{J}(e-a)^{J}$ и $f_{2}=\sum_{J \in \mathbb{Z}_{+}^{n} \backslash \Lambda} x_{J}(e-a)^{J}$ сходятся безусловно в $\mathcal{O}_{\mathfrak{g}}\left(D_{a, r}\right)$, и $f=f_{1}+f_{2}$. Однако $f_{2} \in \mathfrak{S}_{\Lambda}\left(D_{a, r}\right)$, a $f_{1} \in \mathfrak{S}_{\mathbb{Z}_{+}^{n} \backslash \Lambda}\left(D_{a, r}\right)$. Поскольку $\mathcal{O}_{\mathfrak{g}}\left(D_{a, r}\right)$ - пространство Фреше, отсюда вытекает, что $\mathcal{O}_{\mathfrak{g}}\left(D_{a, r}\right)=\mathfrak{S}_{\Lambda}\left(D_{a, r}\right) \oplus \mathfrak{S}_{\mathbb{Z}_{+}^{n} \backslash \Lambda}\left(D_{a, r}\right)$. Следствие доказано.

4.2. Абсолютный базис в $\mathcal{O}_{\mathfrak{g}}$. Пусть $\mathfrak{g}-$ нильпотентная алгебра Ли степени нильпотентности $c, e=\left(e_{1}, \ldots, e_{n}\right)$ - ее треугольный базис, $c^{*}=$ $\max \left\{s: \operatorname{ad}^{s}\left(e_{j}\right)\left(e_{i}\right) \neq 0, i<j\right\},\left\{C_{i j}^{k}\right\}$ - структурные константы алгебры Ли $\mathfrak{g}$ в базисе $e, C=\max \left\{\left|C_{i j}^{k}\right|: i, j, k\right\}$, а $S \subseteq \mathbb{R}_{+}^{n}-$ неограниченно возрастающее подмножество, состоящее из всех наборов $t=\left(t_{1}, \ldots, t_{n}\right)$ таких, что $t_{k} \leqslant t_{i} t_{j}$ всякий раз, когда $d_{\sigma-1}<i \leqslant d_{\sigma}, d_{\tau-1}<j \leqslant d_{\tau}, 1 \leqslant \sigma \leqslant \tau, i<j$ и $k>d_{\tau}$. Тогда $\mathcal{O}_{\mathfrak{g}}=\lim _{\longleftarrow} \mathfrak{A}_{e, S}$ по лемме 4.1 .

Лемма 4.2. Пусть е - треуголъный базис нормального роста в g. Тогда для каждого $t \in S$ найдется $s \in S, s>t$, такой, что

$$
u_{t s}=\sum_{J}\left(s^{-1} t\right)^{J} \alpha_{s, J} \otimes\left(t^{-1} e\right)^{J}, \quad \sum_{J}\left(s^{-1} t\right)^{J}\left\|\alpha_{s, J}\right\|_{\mathcal{A}\left(l_{e, s}\right)}\left\|\left(t^{-1} e\right)^{J}\right\|_{\mathcal{A}\left(l_{e, t}\right)}<\infty,
$$

где $u_{t s}: \mathcal{A}\left(l_{e, s}\right) \rightarrow \mathcal{A}\left(l_{e, t}\right)$ - гомоморфизм алгебр из обратной системы $\mathfrak{A}_{e, S}$, a $\alpha_{s, J}$ - координатный линейный функиионал на $\mathcal{A}\left(l_{e, s}\right)$ относительно слабо однородного базиса $\left\{\left(s^{-1} e\right)^{J}\right\}$.

ДокАЗАтельство. Рассмотрим $f \in \mathcal{A}\left(l_{e, s}\right)$. Подмножество $\left\{\left(s^{-1} e\right)^{J}\right\} \subseteq$ $\mathcal{U}(\mathfrak{g})$ является слабо однородным базисом в $\mathcal{A}\left(l_{e, s}\right)$ (см. предложение 3.1 ), поэтому имеем единственное разложение $f=\sum_{J} \alpha_{s, J}(f)\left(s^{-1} e\right)^{J}$ в формальный степенной ряд. Аналогично, $u_{t s}(f)=\sum_{J} \alpha_{t, J}\left(u_{t s}(f)\right)\left(t^{-1} e\right)^{J}$. Поскольку $u_{t s}$ сводится к тождественному отображению на $\mathcal{U}(\mathfrak{g})$, отсюда вытекает, что $\alpha_{t, J}\left(u_{t s}(f)\right)=\left(s^{-1} t\right)^{J} \alpha_{s, J}(f)$ (см. определение 2.1). Итак,

$$
u_{t s}(f)=\sum_{J}\left(s^{-1} t\right)^{J}\left(\alpha_{s, J} \otimes\left(t^{-1} e\right)^{J}\right)(f) .
$$

Положим $\Gamma_{t s}=\sum_{J} s^{-J}\left\|\alpha_{s, J}\right\|_{\mathcal{A}\left(l_{e, s}\right)}\left\|e^{J}\right\|_{\mathcal{A}\left(l_{e, t}\right)}$. Докажем, что $\Gamma_{t s}<\infty$. По лемме 3.2 имеем

$$
\left\|\alpha_{s, J}\right\|_{\mathcal{A}\left(l_{e, s}\right)} \leqslant \varkappa_{s}^{c^{2}|J|} \prod_{\tau=1}^{c-1} \frac{\left(\left|J_{\tau}\right|+\sum_{\kappa=\tau+1}^{c} c^{*} \wedge(\kappa-\tau)\left|J_{\kappa}\right|\right) !}{\left|J_{\tau}\right| !},
$$

где $\varkappa_{s}=1 \vee\left(c\left(n-d_{1}\right) C_{s}\right), C_{s}=\max \left\{\left|C_{i j}^{k}(s)\right|: i, j, k\right\}$, а $C_{i j}^{k}(s)-$ структурные константы алгебры Ли $\mathfrak{g}$ в базисе $s^{-1} e$. Заметим, что

$$
\left[s_{i}^{-1} e_{i}, s_{j}^{-1} e_{j}\right]=s_{i}^{-1} s_{j}^{-1}\left[e_{i}, e_{j}\right]=\sum_{k>j} s_{k}\left(s_{i} s_{j}\right)^{-1} C_{i j}^{k} s_{k}^{-1} e_{k}
$$


для всех $i, j, i<j$. Учитывая, что $s \in S$, имеем $C_{s} \leqslant \max \left\{s_{k}\left(s_{i} s_{j}\right)^{-1}\left|C_{i j}^{k}\right|\right.$ : $i<j<k\} \leqslant C$. В частности, $\varkappa_{s} \leqslant \varkappa=1 \vee\left(c\left(n-d_{1}\right) C\right)$. По условию рост базиса $e$ нормален. По определению 3.1 имеем $\left\|e_{i}^{k}\right\|_{\mathcal{A}\left(l_{e, t}\right)}^{1 / k} \leqslant \gamma_{i}\left(t_{\mathrm{s}}\right)(1 / k)^{\theta_{i}}$, где $\theta_{i} \geqslant \sum_{\iota=1}^{\tau-1} c^{*} \wedge \iota$ при $e_{i} \in \mathfrak{e}_{\tau}, \tau>1, t_{\mathrm{s}}=\left(t_{1}, \ldots, t_{d_{1}}\right), t_{i}=\left\|e_{i}\right\|_{\mathcal{A}\left(l_{e, t}\right)}$ и $\gamma_{i}(x) \in$ $\mathbb{R}_{+}^{0}\left[x_{1}, \ldots, x_{d_{1}}\right]$. Однако $c^{*} \wedge \iota \leqslant n$ для всех $\iota$. Поэтому $\left(\left(c^{*} \wedge \iota\right) k\right)^{\left(c^{*} \wedge \iota\right)} \leqslant$ $n^{n} k^{\left(c^{*} \wedge \iota\right)}$ и

$$
\begin{aligned}
\prod_{\iota=1}^{\tau-1} \frac{1}{k^{c^{*} \wedge \iota}} & \leqslant n^{(\tau-1) n} \prod_{m=1}^{\tau-1} \frac{1}{\left(\left(c^{*} \wedge m\right) k\right)^{c^{*} \wedge m}} \\
& \leqslant n^{(\tau-1) n} \prod_{m=1}^{\tau-1}\left(\frac{1}{\left(\left(\left(c^{*} \wedge m\right) k\right) !\right)^{1 /\left(c^{*} \wedge m\right) k}}\right)^{c^{*} \wedge m} \\
& =n^{(\tau-1) n}\left(\prod_{m=1}^{\tau-1} \frac{1}{\left(\left(c^{*} \wedge m\right) k\right) !}\right)^{1 / k}
\end{aligned}
$$

Отсюда вытекает, что

$$
\left\|e_{i}^{k}\right\|_{\mathcal{A}\left(l_{e, t}\right)}^{1 / k} \leqslant \gamma_{i}\left(t_{\mathrm{S}}\right) k^{-\sum_{m=1}^{\tau-1} c^{*} \wedge m} \leqslant \gamma_{i}\left(t_{\mathrm{S}}\right) n^{(\tau-1) n}\left(\prod_{m=1}^{\tau-1} \frac{1}{\left(\left(c^{*} \wedge m\right) k\right) !}\right)^{1 / k}
$$

при $d_{\tau-1}<i \leqslant d_{\tau}$. Пользуясь этими оценками, получаем

$$
\begin{aligned}
\left\|e_{\mathrm{r}}^{J_{\mathrm{r}}}\right\|_{\mathcal{A}\left(l_{e, t}\right)} & \leqslant\left\|e_{d_{1}+1}^{j_{d_{1}+1}}\right\|_{\mathcal{A}\left(l_{e, t}\right)} \cdots\left\|e_{n}^{j_{n}}\right\|_{\mathcal{A}\left(l_{e, t}\right)} \\
& \leqslant \gamma\left(t_{\mathrm{s}}\right)^{\left|J_{\mathrm{r}}\right|} \prod_{\tau=2}^{c} \prod_{d_{\tau-1}<i \leqslant d_{\tau}} \prod_{\iota=1}^{\tau-1}\left(\left(\left(c^{*} \wedge \iota\right) j_{i}\right) !\right)^{-1} \\
& =\gamma\left(t_{\mathrm{s}}\right)^{\left|J_{\mathrm{r}}\right|} \prod_{\tau=2}^{c} \prod_{\iota=1}^{\tau-1} \frac{1}{\left(c^{*} \wedge \iota\right) J_{\tau} !}=\gamma\left(t_{\mathrm{s}}\right)^{\left|J_{\mathrm{r}}\right|} \prod_{\tau=1}^{c-1} \prod_{\kappa=\tau+1}^{c} \frac{1}{\left(c^{*} \wedge(\kappa-\tau)\right) J_{\kappa} !},
\end{aligned}
$$

где $\gamma(x)=n^{(c-1) n} \sum_{i>d_{1}} \gamma_{i}(x) \in \mathbb{R}_{+}^{0}\left[x_{1}, \ldots, x_{d_{1}}\right]$. Тогда

$$
\begin{aligned}
\Gamma_{t s} & \leqslant \sum_{J} s^{-J}\left(\gamma\left(t_{\mathrm{s}}\right) \varkappa^{c^{2}}\right)^{|J|} \prod_{\tau=1}^{c-1} \frac{\left(\left|J_{\tau}\right|+\sum_{\kappa=\tau+1}^{c} c^{*} \wedge(\kappa-\tau)\left|J_{\kappa}\right|\right) !}{J_{\tau} ! \prod_{\kappa=\tau+1}^{c}\left(c^{*} \wedge(\kappa-\tau)\right) J_{\kappa} !} \\
& \leqslant \sum_{J} s^{-J}\left(\gamma\left(t_{\mathrm{s}}\right) \varkappa^{c^{2}}\right)^{|J|}(n-1)^{\sum_{\tau=1}^{c-1}\left(\left|J_{\tau}\right|+\sum_{\kappa=\tau+1}^{c} c^{*} \wedge(\kappa-\tau)\left|J_{\kappa}\right|\right)} \\
& \leqslant \sum_{J}\left(s^{-1} \gamma\left(t_{\mathrm{s}}\right) \varkappa^{c^{2}}(n-1)^{c^{*}(c-1)}\right)^{|J|}=\sum_{p=1}^{\infty} p\left(s^{-1} \zeta\left(t_{\mathrm{s}}\right)\right)^{p}<\infty,
\end{aligned}
$$

как только $\zeta\left(t_{\mathrm{s}}\right)=\gamma\left(t_{\mathrm{s}}\right) \varkappa^{c^{2}}(n-1)^{c^{*}(c-1)}<s$. Лемма доказана.

СлеДСТВИЕ 4.2. Алгебра $\mathcal{O}_{\mathfrak{g}}$ является ядерной алгеброй Фреше.

ДокАЗАтЕльство. Для каждого $t \in S$ по лемме 4.2 найдется $s \in S, s>t$, такой, что оператор $u_{t s}: \mathcal{A}\left(l_{e, s}\right) \rightarrow \mathcal{A}\left(l_{e, t}\right)$ является ядерным. Поскольку $\mathcal{O}_{\mathfrak{g}}=$ $\lim _{\mathfrak{L}} \mathfrak{A}_{e, S}$ (лемма 4.1), отсюда вытекает, что $\mathcal{O}_{\mathfrak{g}}$ есть ядерная алгебра Фреше. Следствие доказано. 
Отметим, что следствие 4.2 есть частный случай общего результата, доказанного А. Ю. Пирковским [6, следствие 6.3], о том, что оболочка Аренса-Майкла $\tilde{A}$ любой конечно порожденной ассоциативной алгебры $A$ является ядерной алгеброй Фреше. Поскольку $\mathcal{O}_{F}$ - оболочка Аренса-Майкла алгебры $\mathcal{U}(F)$, получаем, что $\mathcal{O}_{F}$ является ядерной алгеброй Фреше для любой конечномерной алгебры Ли $F$.

Теперь докажем основной результат.

ТЕОРемА 4.1. Пусть е-треуголъный базис нильпотентной алгебры Ли $\mathfrak{g}$, имеющий нормальный рост. Тогда множество всех упорядоченных мономов $\left\{e^{J}\right\}$ является абсолютным базисом в $\mathcal{O}_{\mathfrak{g}}$. Таким образом, если $f=$ $\sum_{J} a_{J} e^{J}-$ формальный степенной ряд в $\mathcal{O}_{\mathfrak{g}}$, то он сходится абсолютно в $\mathcal{O}_{\mathfrak{g}}$.

ДоказАТЕЛьство. Возьмем $f=\sum_{J} a_{J} e^{J} \in \mathcal{O}_{\mathfrak{g}}$. Поскольку $\mathcal{O}_{\mathfrak{g}}=\underset{\lim }{\mathfrak{A}_{e, S}}$ (лемма 4.1), необходимо лишь доказать, что $\sum_{J}\left|a_{J}\right|\left\|e^{J}\right\|_{\mathcal{A}\left(l_{e, t}\right)}<\infty$ для всех $t \in S$. Фиксируем набор $t \in S$. По лемме 4.2 найдется такой набор $s \in S$, что $s>t$ и $\sum_{J} s^{-J}\left\|\alpha_{s, J}\right\|_{\mathcal{A}\left(l_{e, s}\right)}\left\|e^{J}\right\|_{\mathcal{A}\left(l_{e, t}\right)}<\infty$. Если $\chi_{s}: \mathcal{O}_{\mathfrak{g}} \rightarrow \mathcal{A}\left(l_{e, s}\right)-$ каноническая проекция обратной системы $\mathfrak{A}_{e, S}$, то

$$
\begin{aligned}
\sum_{J}\left|a_{J}\right|\left\|e^{J}\right\|_{\mathcal{A}\left(l_{e, t}\right)} & =\sum_{J} s^{-J}\left|\alpha_{s, J}\left(\chi_{s}(f)\right)\right|\left\|e^{J}\right\|_{\mathcal{A}\left(l_{e, t}\right)} \\
& \leqslant \sum_{J} s^{-J}\left\|\alpha_{s, J}\right\|_{\mathcal{A}\left(l_{e, s}\right)}\left\|e^{J}\right\|_{\mathcal{A}\left(l_{e, t}\right)}\left\|\chi_{s}(f)\right\|_{\mathcal{A}\left(l_{e, s}\right)}<\infty,
\end{aligned}
$$

т. е. $\sum_{J}\left|a_{J}\right|\left\|e^{J}\right\|_{\mathcal{A}\left(l_{e, t}\right)}<\infty$. Теорема доказана.

ЗАмЕЧАнИЕ 4.1. То же рассуждение, что и в доказательстве теоремы 4.1, дает, что множество $\left\{(e-a)^{J}\right\}$ всех упорядоченных мономов в $\mathcal{O}_{\mathfrak{g}}\left(D_{a, r}\right)$ также будет абсолютным базисом, но лишь локально. А именно, выберем достаточно малый полирадиус $r^{\prime}<r$ так, что $\zeta\left(r_{\mathrm{s}}^{\prime}\right)=\gamma\left(r_{\mathrm{s}}^{\prime}\right) \varkappa^{c^{2}}(n-1)^{c^{*}(c-1)-1}<r$ (см. доказательство леммы 4.2). Покажем, что $\gamma\left(x^{\prime}\right)=n^{(c-1) n} \sum_{i>d_{1}} \gamma_{i}\left(x^{\prime}\right) \rightarrow 0$ при $x^{\prime} \rightarrow 0$. Тогда для всех $t<r^{\prime}$ имеем $\zeta\left(t_{\mathrm{s}}\right) \leqslant \zeta\left(r_{\mathrm{s}}^{\prime}\right)<r$. Следовательно, каждый формальный степенной ряд $f=\sum_{J} x_{J}(e-a)^{J}$ в $\mathcal{O}_{\mathfrak{g}}\left(D_{a, r}\right)$ сходится абсолютно в $\mathcal{O}_{\mathfrak{g}}\left(D_{a, r^{\prime}}\right)$.

СЛЕДСТВИЕ 4.3. Пусть $\mathfrak{g}$ - нильпотентная алгебра Ли с базисом Кляйнеке-Широкова е. Тогда $\left\{e^{J}\right\} \subseteq \mathcal{U}(\mathfrak{g})-$ абсолютнъй базис в $\mathcal{O}_{\mathfrak{g}}$.

ДокАЗАтЕЛьСтво. Достаточно применить предложение 3.2 и теорему 4.1.

4.3. Семейство норм, задающее топологию пространства $\mathcal{O}_{\mathfrak{g}}$. Пусть $\mathfrak{g}$ - нильпотентная алгебра Ли с треугольным базисом $e=\left(e_{1}, \ldots, e_{n}\right)$, и пусть $S \subseteq \mathbb{R}_{+}^{n}$ - неограниченно возрастающее подмножество, введенное в п. 4.2. Возьмем $f \in \mathcal{O}_{\mathfrak{g}}$. По предложению 4.1 имеем разложение $f=\sum_{J} a_{J} e^{J}$ в формальный степенной ряд. Введем полунормы

$$
p_{t}\left(\sum_{J} a_{J} e^{J}\right)=\sum_{J}\left|a_{J}\right| w_{J} t^{J} \quad \text { на } \quad \mathcal{O}_{\mathfrak{g}},
$$


где $t \in S, t^{J}=t_{1}^{j_{1}} \cdots t_{n}^{j_{n}}$, а $w_{J}-$ веса, заданные формулой

$$
w_{J}=\varkappa^{-c^{2}|J|} \prod_{\tau=1}^{c-1} \frac{J_{\tau} !}{\left(\left|J_{\tau}\right|+\sum_{\kappa=\tau+1}^{c} c^{*} \wedge(\kappa-\tau)\left|J_{\kappa}\right|\right) !} .
$$

Докажем две технические леммы.

ЛЕмма 4.3. Пусть $f=\sum_{J} a_{J} e^{J}-$ разложение произвольного элемента $f \in \mathcal{O}_{\mathfrak{g}}$. Тогда $p_{t}(f) \leqslant \zeta_{t s}\|f\|_{\mathcal{A}\left(l_{e, s}\right)}$, где $t, s \in S, t<s$, a $\zeta_{t s}-$ положительная константа, зависящая от s ut.

ДокАЗАТЕЛЬСтво. По лемме 3.2 имеем

$$
\left|a_{J} s^{J}\right|=\left|\alpha_{s, J}(f)\right| \leqslant \varkappa^{c^{2}|J|} \prod_{\tau=1}^{c-1} \frac{\left(\left|J_{\tau}\right|+\sum_{\kappa=\tau+1}^{c} c^{*} \wedge(\kappa-\tau)\left|J_{\kappa}\right|\right) !}{J_{\tau} !}\|f\|_{\mathcal{A}\left(l_{e, s}\right)},
$$

где $f=\sum_{J} a_{J} s^{J}\left(s^{-1} e\right)^{J} \in \mathcal{A}\left(l_{e, s}\right)$, т. е. $\left|a_{J} s^{J}\right| \leqslant w_{J}^{-1}\|f\|_{\mathcal{A}\left(l_{e, s}\right)}$. Тогда

$$
p_{t}(f)=\sum_{J}\left|a_{J}\right| s^{J} w_{J}\left(s^{-1} t\right)^{J} \leqslant \sum_{J}\|f\|_{\mathcal{A}\left(l_{e, s}\right)}\left(s^{-1} t\right)^{J} \leqslant\|f\|_{\mathcal{A}\left(l_{e, s}\right)} \zeta_{t s}
$$

где $\zeta_{t s}=\sum_{k=1}^{\infty} k\left(\max \left(s^{-1} t\right)\right)^{k}$. Лемма доказана.

Лемма 4.4. Допустим, что рост базиса е нормален, и пусть $t \in S$. Тогда существует набор $s \in S, s>t$, такой, что $\|f\|_{\mathcal{A}\left(l_{e, t}\right)} \leqslant p_{s}(f)$ для всех $f \in \mathcal{O}_{\mathfrak{g}}$.

ДокАЗАТЕЛЬство. Рассмотрим $f \in \mathcal{O}_{\mathfrak{g}}$. Тогда по теореме 4.1 степенной ряд $f=\sum_{J} a_{J} e^{J}$ сходится абсолютно в $\mathcal{O}_{\mathfrak{g}}$. Следовательно,

$$
\begin{aligned}
\|f\|_{\mathcal{A}\left(l_{e, t}\right)} & \leqslant \sum_{J}\left|a_{J}\right|\left\|e^{J}\right\|_{\mathcal{A}\left(l_{e, t}\right)}=\sum_{J}\left|a_{J}\right| s^{J} w_{J} s^{-J} w_{J}^{-1}\left\|e^{J}\right\|_{\mathcal{A}\left(l_{e, t}\right)} \\
& \leqslant p_{s}(f) \sup _{J}\left\{s^{-J} w_{J}^{-1}\left\|e^{J}\right\|_{\mathcal{A}\left(l_{e, t}\right)}\right\} .
\end{aligned}
$$

По условию базис $е$ имеет нормальный рост. Рассуждая, как в доказательстве леммы 4.2, получаем

$$
\begin{aligned}
\left\|e^{J}\right\|_{\mathcal{A}\left(l_{e, t}\right)} & \leqslant\left\|e_{\mathrm{s}}^{J_{\mathrm{s}}}\right\|_{\mathcal{A}\left(l_{e, t}\right)}\left\|e_{\mathrm{r}}^{J_{\mathrm{r}}}\right\|_{\mathcal{A}\left(l_{e, t}\right)} \leqslant t_{\mathrm{s}}^{J_{\mathrm{s}}}\left\|e_{\mathrm{r}}^{J_{\mathrm{r}}}\right\|_{\mathcal{A}\left(l_{e, t}\right)} \\
& \leqslant \delta\left(t_{\mathrm{s}}\right)^{|J|} \prod_{\tau=1}^{c-1} \prod_{\kappa=\tau+1}^{c} \frac{1}{c^{*} \wedge(\kappa-\tau) J_{\kappa} !},
\end{aligned}
$$

где $\delta(x)=\gamma(x) \sum_{i=1}^{d_{1}} x_{i} \in \mathbb{R}_{+}^{0}\left[x_{1}, \ldots, x_{d_{1}}\right]$. Отсюда вытекает, что

$$
\begin{aligned}
\|f\|_{\mathcal{A}\left(l_{e, t}\right)} & \leqslant p_{s}(f) \sup _{J}\left\{\left(s^{-1} \delta\left(t_{\mathrm{s}}\right) \varkappa^{c^{2}}\right)|J| \prod_{\tau=1}^{c-1} \frac{\left(\left|J_{\tau}\right|+\sum_{\kappa=\tau+1}^{c} c^{*} \wedge(\kappa-\tau)\left|J_{\kappa}\right|\right) !}{J_{\tau} ! \prod_{\kappa=\tau+1}^{c} c^{*} \wedge(\kappa-\tau) J_{\kappa} !}\right\} \\
& \leqslant p_{s}(f) \sup _{J}\left\{\left(s^{-1} \delta\left(t_{\mathrm{s}}\right) \varkappa^{c^{2}}(n-1)^{c^{*}(c-1)}\right)^{|J|}\right\} .
\end{aligned}
$$

Поэтому если $\delta\left(t_{\mathrm{s}}\right) \varkappa^{c^{2}}(n-1)^{c^{*}(c-1)} \leqslant s$, то $\|f\|_{\mathcal{A}\left(l_{e, t}\right)} \leqslant p_{s}(f)$. Лемма доказана. 
ТеОремА 4.2. Пусть $\mathfrak{g}$ - нильпотентная алгебра Ли с треугольным базисом е нормалъного роста. Тогда семейство норм $\left\{p_{t}: t \in S\right\}$ задает исходную топологию пространства $\mathcal{O}_{\mathfrak{g}}$.

ДокАЗАТЕЛЬСтво. По лемме 4.1 топология пространства $\mathcal{O}_{\mathfrak{g}}$ задается семейством $\left\{\|\cdot\|_{\mathcal{A}\left(l_{e, t}\right)}: t \in S\right\}$ норм на $\mathcal{U}(\mathfrak{g})$. Фиксируем набор $t \in S$. Тогда по лемме 4.4 имеем $\|\cdot\|_{\mathcal{A}\left(l_{e, t}\right)} \leqslant p_{s}$ для некоторого $s \in S, s>t$. С другой стороны, с помощью леммы 4.3 мы выводим, что $p_{t} \leqslant \zeta_{t s}\|\cdot\|_{\mathcal{A}\left(l_{e, s}\right)}$ для всех $t, s \in S, t<s$. Следовательно, $\left\{\|\cdot\|_{\mathcal{A}\left(l_{e, t}\right)}: t \in S\right\}$ и $\left\{p_{t}: t \in S\right\}$ - эквивалентные семейства норм на $\mathcal{O}_{\mathfrak{g}}$. Теорема доказана.

4.4. Частные производные. Чтобы получить явные выражения для коэффициентов формального степенного ряда $f=\sum_{J} a_{J} e^{J} \in \mathcal{O}_{\mathfrak{g}}$, мы исследуем в этом пункте вопрос о непрерывности частных производных $\partial_{e_{i}} \in \mathcal{L}(\mathcal{U}(F))$, $\partial_{e_{i}}\left(e_{1}^{j_{1}} \cdots e_{n}^{j_{n}}\right)=j_{i} e_{1}^{j_{1}} \cdots e_{i}^{j_{i}-1} \cdots e_{n}^{j_{n}}$ относительно базиса $e$. Сначала рассмотрим случай градуированной нильпотентной алгебры Ли. Пусть $\mathfrak{F}_{x}-$ конечномерная $\left(n=\operatorname{dim}\left(\mathfrak{F}_{x}\right)\right)$ градуированная (натуральными числами $\left.1,2, \ldots, c\right)$ нильпотентная алгебра Ли со свободными образующими Ли $x=\left(x_{1}, \ldots, x_{m}\right)$. Иными словами, $\mathfrak{F}_{x}$ - факторалгебра свободной алгебры Ли, порожденной $x$, по $(c+1)$-й степени ее нижнего центрального ряда. Таким образом, $\mathfrak{F}_{x}=$ $\bigoplus_{i=1}^{c} \mathfrak{F}_{i}(x)$, где $\mathfrak{F}_{i}(x)$ - подпространство в $\mathfrak{F}_{x}$, порожденное лиевыми словами длины $i$ от образующих, входящих в $x$. Кроме того, $\mathfrak{F}_{x}^{(k)}=\bigoplus_{i \geqslant k} \mathfrak{F}_{i}(x)$ и $\mathfrak{F}_{x}^{(c+1)}=\{0\}$.

Возьмем в $\mathfrak{F}_{x}$ любой треугольный базис $y=\left(y_{1}, \ldots, y_{n}\right)$ с первыми $m$ элементами $y_{i}=x_{i}, 1 \leqslant i \leqslant m$. Заметим, что операторы частного дифференцирования $\partial_{x_{i}} \in \mathcal{L}\left(\mathcal{U}\left(\mathfrak{F}_{x}\right)\right), 1 \leqslant i \leqslant m($ см. $\S 2)$, не зависят от выбора треугольного базиса с первыми $m$ элементами $x_{1}, \ldots, x_{m}$.

ЛЕмма 4.5. Пусть $\mathfrak{F}_{x}-$ градуированная нильпотентная алгебра Ли со свободными образующими Ли $x=\left(x_{1}, \ldots, x_{m}\right)$. Тогда все операторы $\partial_{x_{i}} \in \mathcal{L}\left(\mathcal{U}\left(\mathfrak{F}_{x}\right)\right)$ допускают непрерывные продолжения до операторов $\partial_{x_{i}} \in \mathcal{L}\left(\mathcal{O}_{\mathfrak{F}_{x}}\right)$ соответственно.

ДокАЗАТЕЛЬСтво. Фиксируем $i, j, 1 \leqslant i<j \leqslant m$. Тогда семейство

$$
\left(x_{j},\left[x_{j}, x_{i}\right], x_{1}, \ldots, \widehat{x}_{j}, \ldots, x_{m},\left[x_{p}, x_{q}\right]: p<q,(p, q) \neq(i, j)\right) \cup \varsigma
$$

является базисом в $\mathfrak{F}_{x}$, где $\varsigma$ - часть базиса $y$ в $\mathfrak{F}_{x}^{(3)}$. Каждый оператор ad $v$, $v \in \mathfrak{F}_{x}$, присоединенного представления алгебры $\mathfrak{F}_{x}$ задается в этом базисе строго нижнетреугольной $(n \times n)$-матрицей $\mathcal{C}_{v}=\left(a_{i j}^{v}\right)_{i, j}$. Если $v=y_{k}$, то положим $\mathcal{C}_{k}=\mathcal{C}_{v}=\left(a_{i j}^{k}\right)_{i, j}, 1 \leqslant k \leqslant n$. Ясно, что $a_{21}^{i}=-1$ и $a_{21}^{k}=0$ для всех $k \neq i$. Возьмем неограниченно возрастающее подмножество $S$ в $\mathbb{R}_{+}^{n}$. Тогда $\mathcal{O}_{\mathfrak{F}}=\lim _{\mathfrak{y}, S}$ по лемме 4.1, где $\mathfrak{A}_{y, S}=\left\{\mathcal{A}\left(l_{y, s}\right), u_{t s}, s, t \in S\right\}$ - проективная система. Фиксируем набор $t \in S$ и положим $\overline{\mathcal{C}}_{v}=\left(a_{i j}^{v} \cdot 1_{\mathcal{A}\left(l_{y, t}\right)}\right)_{i, j} \in \mathcal{M}_{n}\left(\mathcal{A}\left(l_{y, t}\right)\right), v \in \mathfrak{F}_{x}$. Зададим представление алгебр Ли $\theta: \mathfrak{F}_{x} \rightarrow \mathcal{M}_{n}\left(\mathcal{A}\left(l_{y, t}\right)\right)$ формулой $\theta(v)=\operatorname{diag}(v)-\overline{\mathcal{C}}_{v}^{*}$, в которой $\operatorname{diag}(v)$ - диагональная $(n \times n)$-матрица с диагональными элементами $v$, а $\overline{\mathcal{C}}_{v}^{*}-$ матрица, транспонированная к $\overline{\mathcal{C}}_{v}$ (см. [16, §7]). Заметим, что $\gamma_{12}^{i}=1_{\mathcal{A}\left(l_{y, t}\right)}$ и $\gamma_{12}^{k}=0$ для всех $k \neq i$, где $\left(\gamma_{i j}^{k}\right)_{i, j}=\theta\left(y_{k}\right)=\operatorname{diag}\left(y_{k}\right)-\overline{\mathcal{C}}_{k}^{*}$. Поскольку $\mathcal{M}_{n}\left(\mathcal{A}\left(l_{y, t}\right)\right)$ - банахова алгебра и $\mathcal{O}_{\mathfrak{F}_{x}}$ - оболочка Аренса-Майкла 
алгебры $\mathcal{U}\left(\mathfrak{F}_{x}\right)$, мы видим, что $\theta$ имеет непрерывное продолжение до гомоморфизма алгебр $\theta: \mathcal{O}_{\mathfrak{F}_{x}} \rightarrow \mathcal{M}_{n}\left(\mathcal{A}\left(l_{y, t}\right)\right)$. Положим $\theta\left(y^{J}\right)=\left(\gamma_{i j}^{(J)}\right)_{i, j}$, где $y^{J}=y_{1}^{j_{1}} \cdots y_{n}^{j_{n}}-$ упорядоченный моном в $\mathcal{U}\left(\mathfrak{F}_{x}\right)$ от элементов базиса $y$. Легко проверяется, что $\gamma_{12}^{(J)}=j_{i} y_{1}^{j_{1}} \cdots y_{i}^{j_{i}-1} \cdots y_{n}^{j_{n}}$. Если $P_{12}: \mathcal{M}_{n}\left(\mathcal{A}\left(l_{y, t}\right)\right) \rightarrow \mathcal{A}\left(l_{y, t}\right)-$ проекция, $P_{12}\left(a_{l v}\right)_{l, v}=a_{12}$, то $\partial_{x_{i}}=P_{12} \theta$, т. е. $\partial_{x_{i}}$ продолжается до непрерывного оператора $\partial_{x_{i}}: \mathcal{O}_{\mathfrak{F}_{x}} \rightarrow \mathcal{A}\left(l_{y, t}\right)$. Поскольку это верно для всех наборов $t \in S$, мы заключаем, что оператор $\partial_{x_{i}}$ непрерывен относительно топологии пространства $\mathcal{O}_{\mathfrak{F}_{x}}$. Лемма доказана.

ПреДЛОЖЕНИЕ 4.2. Пусть $\mathfrak{g}$ - нильпотентная алгебра Ли с таким треугольным базисом $е=\left(e_{1}, \ldots, e_{n}\right)$, что $\left(e_{m+1}, \ldots, e_{n}\right)$ является базисом в $[\mathfrak{g}, \mathfrak{g}]$. Тогда операторы $\partial_{e_{i}} \in \mathcal{L}(\mathcal{U}(\mathfrak{g}))$ допускают непрерывные продолжения до операторов $\partial_{e_{i}} \in \mathcal{L}\left(\mathcal{O}_{\mathfrak{g}}\right)$ соответственно, $1 \leqslant i \leqslant m$.

ДокАЗАТЕЛЬСтво. Пусть $\mathfrak{g}^{(c+1)}=\{0\}$, и пусть $\mathfrak{F}_{x}-$ градуированная (натуральными числами $1,2, \ldots, c)$ нильпотентная алгебра Ли со свободными образующими Ли $x=\left(x_{1}, \ldots, x_{m}\right)$. Тогда существует эпиморфизм алгебр Ли $\tau: \mathfrak{F}_{x} \rightarrow \mathfrak{g}$ такой, что $\tau\left(x_{i}\right)=e_{i}$ для всех $i, 1 \leqslant i \leqslant m$. Можно дополнить $x$ до такого треугольного базиса $y$ алгебры Ли $\mathfrak{F}_{x}$, что $\tau\left(y_{i_{k}}\right)=e_{k}$ при некотором $i_{k}$, $i_{k}>m, m<k \leqslant n$, и $y_{s} \in \operatorname{ker}(\tau)$ для всех $s \neq i_{k}, s>m$ (см. подробно об этом [9]). По лемме 4.5 существуют операторы $\partial_{x_{i}} \in \mathcal{L}\left(\mathcal{O}_{\mathfrak{F}}\right), 1 \leqslant i \leqslant m$, продолжающие операторы частного дифференцирования относительно базиса $y$.

Далее, благодаря эпиморфизму $\tau$ имеем отождествление $\mathcal{O}_{\mathfrak{F}} / \mathcal{R}_{\operatorname{ker}(\tau)}=\mathcal{O}_{\mathfrak{g}}$ (см. [6], [9]), где $\mathcal{R}_{\operatorname{ker}(\tau)}$ - замыкание в $\mathcal{U}(F)$ идеала $\mathcal{J}_{\operatorname{ker}(\tau)}$, порожденного идеалом Ли $\operatorname{ker}(\tau)$. Легко видеть, что $\partial_{x_{i}}\left(\mathcal{J}_{\operatorname{ker}(\tau)}\right) \subseteq \mathcal{J}_{\operatorname{ker}(\tau)}$ для всех $i$, $1 \leqslant i \leqslant m$. Поэтому все операторы $\partial_{x_{i}}, 1 \leqslant i \leqslant m$, также оставляют инвариантным идеал $\mathcal{R}_{\operatorname{ker}(\tau)}$, следовательно, корректно определены фактороператоры $\partial_{x_{i}}^{\sim} \in \mathcal{L}\left(\mathcal{O}_{F} / \mathcal{R}_{\operatorname{ker}(\tau)}\right)$. Остается заметить, что $\partial_{x_{i}}^{\sim}=\partial_{e_{i}}$ для всех $i, 1 \leqslant i \leqslant m$. Предложение доказано.

Если $e$ - треугольный базис нормального роста, то предложение 4.2 допускает следующее усиление.

Теорема 4.3. Пусть $\mathfrak{g}$ - нильпотентная алгебра Ли с треугольным базисом $е=\left(e_{1}, \ldots, e_{n}\right)$ нормального роста. Тогда все операторы $\partial_{e_{i}} \in \mathcal{L}(\mathcal{U}(\mathfrak{g}))$ допускают непрерывное продолжение до операторов $\partial_{e_{i}} \in \mathcal{L}\left(\mathcal{O}_{\mathfrak{g}}\right)$ соответственно, $1 \leqslant i \leqslant n$.

ДокАЗАТЕльСтво. В силу предложения 4.2 достаточно доказать это утверждение для операторов $\partial_{e_{i}}, i>m$. Пользуясь теоремой 4.2 , заключаем, что топология пространства $\mathcal{O}_{\mathfrak{g}}$ задается семейством норм $\left\{p_{t}: t \in S\right\}$. Возьмем $i$ так, что $d_{\sigma-1}<i \leqslant d_{\sigma}$ для некоторого $\sigma>1$, и рассмотрим разложение $f=\sum_{J} a_{J} e^{J}$ произвольного элемента $f \in \mathcal{U}(\mathfrak{g})$. Тогда

$$
\begin{aligned}
p_{t}\left(\partial_{e_{i}} f\right) \leqslant \sum_{J} & j_{i}\left|a_{J}\right| \varkappa^{-c^{2}(|J|-1)} t_{1}^{j_{1}} \cdots t_{i}^{j_{i}-1} \cdots t_{n}^{j_{n}} \\
& \times \prod_{\tau=1}^{\sigma-1} \frac{J_{\tau} !}{\left(\left|J_{\tau}\right|-c^{*}+\sum_{\kappa=\tau+1}^{c} c^{*} \wedge(\kappa-\tau)\left|J_{\kappa}\right|\right) !}
\end{aligned}
$$




$$
\begin{aligned}
& \times \frac{j_{d_{\sigma-1}} ! \cdots\left(j_{i}-1\right) ! \cdots j_{d_{\sigma}} !}{\left(\left|J_{\sigma}\right|-1+\sum_{\kappa=\sigma+1}^{c} c^{*} \wedge(\kappa-\tau)\left|J_{\kappa}\right|\right) !} \\
& \times \prod_{\tau=\sigma+1}^{c-1} \frac{J_{\tau} !}{\left(\left|J_{\tau}\right|+\sum_{\kappa=\tau+1}^{c} c^{*} \wedge(\kappa-\tau)\left|J_{\kappa}\right|\right) !} \\
&\left.\leqslant \varkappa^{c^{2}} t_{i}^{-1} \sum_{J}\left|a_{J}\right| s^{J} w_{J}\left(s^{-1} t\right)^{J} \prod_{\tau=1}^{\sigma}\left(\left|J_{\tau}\right|+\sum_{\kappa=\tau+1}^{c} c^{*} \wedge(\kappa-\tau)\left|J_{\kappa}\right|\right)\right)^{c} \\
& \leqslant \varkappa^{c^{2}} t_{i}^{-1} c^{\sigma c} p_{s}(f) \sup _{J}\left\{|J|^{\sigma c} \delta_{s, t}^{|J|}\right\}=\varkappa^{c^{2}} t_{i}^{-1} c^{\sigma c} \sup _{k}\left\{k^{\sigma c} \delta_{s, t}^{k}\right\} p_{s}(f),
\end{aligned}
$$

где $t, s \in S, t<s$, и $\delta_{s, t}=\max \left(s^{-1} t\right)$. Таким образом, $p_{t}\left(\partial_{e_{i}} f\right) \leqslant M_{s, t, \sigma} p_{s}(f)$ для некоторой положительной константы $M_{s, t, \sigma}$ и всех $f \in \mathcal{U}(\mathfrak{g})$. Отсюда вытекает, что $\partial_{e_{i}}-$ непрерывный оператор на $\mathcal{U}(\mathfrak{g})$ относительно топологии, порожденной семейством норм $\left\{p_{s}: s \in S\right\}$. Поскольку это же семейство норм задает топологию пространства $\mathcal{O}_{\mathfrak{g}}$, мы заключаем, что $\partial_{e_{i}} \in \mathcal{L}\left(\mathcal{O}_{\mathfrak{g}}\right)$. Теорема доказана.

СлЕДСТВИЕ 4.4. Пусть $\mathfrak{g}$ - нильпотентная алгебра Ли с треугольным базисом е нормального роста, и пусть $f \in \mathcal{O}_{\mathfrak{g}}$. Тогда ряд

$$
f=\sum_{J} \frac{\left(\partial_{e}^{|J|} f\right)(0)}{J !} e^{J}
$$

является абсолютно сходящимся разложением элемента $f$ в пространстве $\mathcal{O}_{\mathfrak{g}}$, где $\partial_{e}^{|J|}=\partial_{e_{1}}^{j_{1}} \cdots \partial_{e_{n}}^{j_{n}}$.

Наличие абсолютного базиса в $\mathcal{O}_{\mathfrak{g}}$ влечет за собой существование тонкой связи между некоммутативной спектральной теорией и некоммутативной теорией функций [11] (см. также [17]). А именно, если нильпотентная алгебра Ли $\mathfrak{g}$ имеет нормальный рост, то вложение $\mathcal{U}(\mathfrak{g}) \rightarrow \mathcal{O}_{\mathfrak{g}}$ является локализацией (см. [10]). Тем самым, для произвольного левого банахова $\mathcal{O}_{\mathfrak{g}}$-модуля $X$ условие $\mathbb{C}(\lambda) \perp X$ (т. е. $\operatorname{Tor}_{k}^{\mathcal{O}_{\mathfrak{g}}}(\mathbb{C}(\lambda), X)=\{0\}$ для всех $k$ ) эквивалентно тому, что $\lambda$ принадлежит резольвентному множеству относительно спектра Тэйлора $\mathfrak{g}$-модуля $X$ [3], где $\mathbb{C}(\lambda)$ - одномерный правый $\mathcal{O}_{\mathfrak{g}}$-модуль, ассоциированный с характером $\lambda$.

\section{Список литературы}

1. J. L. Taylor, "A general framework for a multi-operator functional calculus", Advances in Math., 9:2 (1972), 183-252.

2. А. А. Досиев, "Когомологии пучков алгебр Фреше и спектральная теория", Функи. анализ и его прил., 39:3 (2005), 76-80; англ. пер.: А. A. Dosiev, "Cohomology of sheaves of Frechet algebras and spectral theory", Funct. Anal. Appl., 39:3 (2005), $225-228$.

3. A. Dosiev, "Cartan-Slodkowski spectra, splitting elements and noncommutative spectral mapping theorems", J. Funct. Anal., 230:2 (2006), 446-493.

4. J. Eschmeier, M. Putinar, Spectral decompositions and analytic sheaves, London Math. Soc. Monogr. (N.S.), 10, Clarendon Press, Oxford Univ. Press, Oxford, 1996.

5. А.Я. Хелемский, Гомология в банаховых и топологических алгебрах, Изд-во Моск. ун-та, М., 1986; англ. пер.: А. Ya. Khelemskii, The homology of Banach and topological algebras, Math. Appl. (Soviet Ser.), 41, Kluwer Acad. Publ., Dordrecht, 1989. 
6. A. Yu. Pirkovskii, "Stably flat completions of universal enveloping algebras", Dissertationes Math. (Rozprawy Mat.), 441 (2006), 5-56; arXiv: math/0311492v2.

7. R. Meyer, Embeddings of derived categories of bornological modules, arXiv: math/0410596v1.

8. А.А. Досиев, "Голоморфные функции от базиса нильпотентной алгебры Ли", Функи. анализ и его прил., 34:4 (2000), 82-84; англ. пер.: А. A. Dosiev, "Holomorphic functions of a basis of a nilpotent Lie algebra", Funct. Anal. Appl., 34:4 (2000), 302-304.

9. А.А. Досиев, “Алгебры степенных рядов от элементов алгебры Ли и спектры Слодковского", Исследования по линейным операторам и теории функиий. 30, Зап. научн. сем. ПОМИ, 290, ПОМИ, СПб., 2002, 72-121; англ. пер.: А. A. Dosiev, "Algebras of power series of elements of a Lie algebra and the Slodkowski spectra", $J$. Math. Sci. (N. Y.), 124:2 (2004), 4886-4908.

10. А. А. Досиев, "Гомологические размерности алгебры целых функций от элементов нильпотентной алгебры Ли", Функи. анализ и его прил., 37:1 (2003), 73-77; англ. пер.: A. A. Dosiev, "Homological dimensions of the algebra formed by entire functions of elements of a nilpotent Lie algebra", Funct. Anal. Appl., 37:1 (2003), 61-64.

11. A. A. Dosiev, "Local left invertibility for operator tuples and noncommutative localizations", J. K-Theory, 4:1 (2009), 163-191.

12. А.Я. Хелемский, Банаховы и полинормированнъе алгебры: общая теория, представления, гомология, Наука, М., 1989.

13. Ж. Диксмье, Универсальные обертывающие алгебры, Мир, М., 1978; пер. с фр.: J. Dixmier, Algèbres enveloppantes, Gauthier-Villars, Paris-Brussels-Montreal, 1974.

14. Ю.В. Туровский, "О коммутативности по модулю радикала Джекобсона ассоциативной оболочки алгебры Ли", Спектральная теория операторов и ее приложения, 8, Баку, 1987, 199-211.

15. Ю.В. Туровский, "Спектральные свойства элементов нормированных алгебр и инвариантные подпространства", Функи. анализ и его прил., 18:2 (1984), 77-78; англ. пер.: Yu. V. Turovskii, "Spectral properties of elements of normed algebras and invariant subspaces", Funct. Anal. Appl., 18:2 (1984), 152-154.

16. A. A. Dosiev, "Spectra of infinite parametrized Banach complexes", J. Operator Theory, 48:3 (2002), 585-614.

17. A. A. Dosi, "Formally-radical functions in elements of a nilpotent Lie algebra and noncommutative localizations", Algebra Colloq. (to appear).

А. А. Доси (А. A. Dosi (Dosiev))

Поступило в редакцию

Middle East Technical University Northern Cyprus Campus,

10.05.2007

Turkey

E-mail: dosiev@yahoo.com

Перевод с англ. А. В. Домрина 